\title{
Rational engineering of Kluyveromyces marxianus to create a chassis for the production of aromatic products
}

\author{
Arun S. Rajkumar and John P. Morrissey ${ }^{*}$ (1)
}

\begin{abstract}
Background: The yeast Kluyveromyces marxianus offers unique potential for industrial biotechnology because of useful features like rapid growth, thermotolerance and a wide substrate range. As an emerging alternative platform, $K$. marxianus requires the development and validation of metabolic engineering strategies to best utilise its metabolism as a basis for bio-based production.

Results: To illustrate the synthetic biology strategies to be followed and showcase its potential, we describe a comprehensive approach to rationally engineer a metabolic pathway in K. marxianus. We use the phenylalanine biosynthetic pathway both as a prototype and because phenylalanine is a precursor for commercially valuable secondary metabolites. First, we modify and overexpress the pathway to be resistant to feedback inhibition so as to overproduce phenylalanine de novo from synthetic minimal medium. Second, we assess native and heterologous means to increase precursor supply to the biosynthetic pathway. Finally, we eliminate branch points and competing reactions in the pathway and rebalance precursors to redirect metabolic flux to a specific product, 2-phenylethanol (2-PE). As a result, we are able to construct robust strains capable of producing over $800 \mathrm{mg} \mathrm{L}^{-1}$ 2-PE from minimal medium.
\end{abstract}

Conclusions: The strains we constructed are a promising platform for the production of aromatic amino acid-based biochemicals, and our results illustrate challenges with attempting to combine individually beneficial modifications in an integrated platform.

Keywords: 2-phenylethanol, Aromatic amino acid, Shikimate pathway, Pentose phosphate pathway, Phosphoketolase, PEP synthase

\section{Introduction}

Microbial cell factories are an important part of an emerging sustainable bioeconomy. By metabolically engineering microbial hosts, it is possible to synthesise chemical compounds that are currently sourced from non-renewable resources or from renewable resources that may not meet the growing demands of the chemical, food and pharmaceutical industries. The process typically

*Correspondence: j.morrissey@ucc.ie

School of Microbiology, Centre for Synthetic Biology and Biotechnology, Environmental Research Institute, APC Microbiome Institute, University

College Cork, Cork T12 K8AF, Ireland involves cloning heterologous pathways for such compounds into host microbes and altering native pathways to ensure that metabolism is optimised for the products of interest. The budding yeast Saccharomyces cerevisiae has proved to be an excellent host for de novo synthesis of valuable secondary metabolites in the flavonoid, stilbenoid and alkaloid families [1]. Several of these are directly derived from aromatic amino acids: flavonoids, stilbenoids and aroma compounds from phenylalanine [2]; benzylisquinoline alkaloids from tyrosine [3]; and monoterpene alkaloids from tryptophan [4]. Others are derived from intermediates of the shikimate pathway, such as the flavour compound vanillin [5] and the fine 
chemical muconic acid [6]. Optimising precursor supply is of special interest if these compounds are to be produced at titres for commercial production competitive with existing commercial sources. As a result, the metabolic engineering of S. cerevisiae to overproduce aromatic amino acids (AAAs) has been the subject of considerable research and development to produce diverse secondary metabolites of commercial value [1, 7]. Furthermore, phenylalanine and tyrosine are commercial products in themselves; at present they are among the few amino acids that are not predominantly industrially produced by bacterial fermentation on an industrial scale [8].

In yeast, AAA synthesis begins with the shikimate pathway (Fig. 1), wherein the DAHP synthases Aro3p and Aro4p convert erythrose-4-phosphate (E4P) and phosphoenolpyruvate (PEP) to 3-deoxy-D-arabinoheptulosonate-7-phosphate (DAHP). This is in turn converted to chorismate via Aro1p and Aro2p. Chorismate is then either committed to phenylalanine/tyrosine biosynthesis via conversion to prephenate by Aro7p, or to tryptophan biosynthesis via conversion to anthranilate by Trp2p/Trp3p. In the former case, the enzymes Pha2p or Tyr1p convert prephenate to phenyl- or hydroxyphenyl-pyruvate, which are then transaminated to phenylalanine or tyrosine respectively by Aro8p and Aro9p. In native biosynthetic pathways, Aro3p, Aro4p and Aro7p are feedback inhibited by tyrosine and/or phenylalanine as committing to this pathway is metabolically expensive. To overcome this limitation, feedback-resistant alleles of all three genes have been identified and exploited to overproduce AAAs. Typically, overexpressing feedbackresistant (fbr) ARO4 (ARO4 $\left.{ }^{f b r}\right)$ and ARO7 $\left(A R O 7^{f b r}\right)$ is sufficient to overproduce phenylalanine and tyrosine for the production of secondary metabolites [2, 9]. Beyond this, various studies have partially or completely overexpressed the shikimate or phenylalanine/tyrosine pathways to further direct flux down this pathway. For example, E4P supply to the pathway can be increased in $S$. cerevisiae by partially overexpressing the non-oxidative branch of its pentose phosphate pathway (PPP) [6, $10]$ and PEP supply to the shikimate pathway has been increased by enhancing its natural production or slowing its conversion to pyruvate $[11,12]$.

While $S$. cerevisiae remains a favoured host, for several reasons, there is considerable value in exploring alternative yeast platforms for the synthesis of aromatic compounds. First, the native metabolism of $S$. cerevisiae has evolved for ethanol production, often at the expense of other pathways. Thus, the metabolism of a Crabtree-negative yeast can offer a better starting point since carbon flux is not diverted to ethanol and the carbon distribution between the various pathways that give rise to anabolic precursors is better balanced. Second,

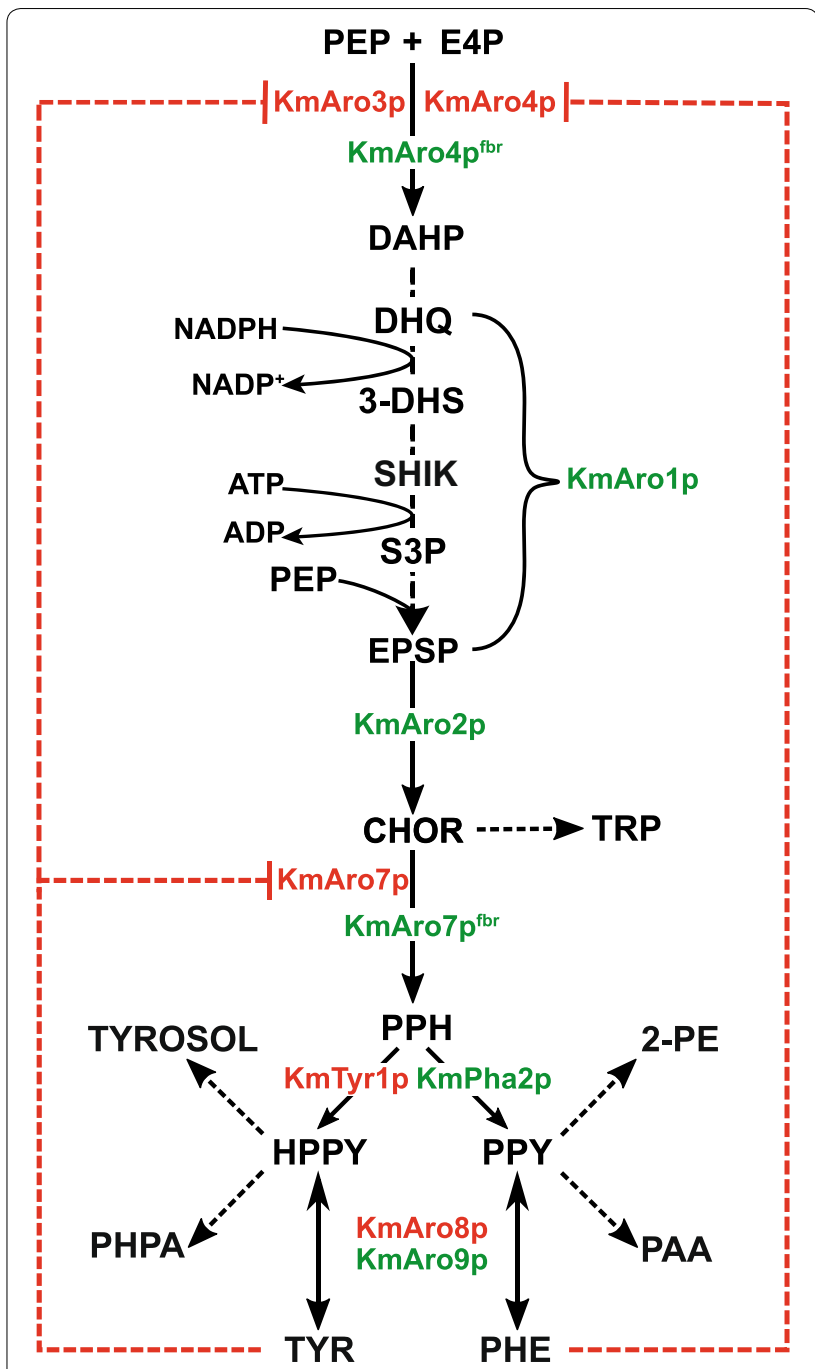

Fig. 1 Shikimate and phenylalanine/tyrosine biosynthesis in Kluyveromyces marxianus. The enzymes are named according to their orthologues in S. cerevisiae. In our study, genes for enzymes coloured red have been deleted or otherwise inactivated and those coloured green are overexpressed, with feedback-resistant alleles represented by the superscript fbr. Red dashed lines indicate product inhibition on enzymes, while dashed arrows indicated multiple reactions between the metabolites indicated. Abbreviations: PEP: phosphoenolpyruvate, E4P: erythrose 4-phosphate, DAHP: 3-deoxy-D-arabinoheptulosonat e-7-phosphate, DHQ: 3-dehydroquinate, 3-DHS: 3-dehydroshikimate, SHIK: shikimic acid, S3P: shikimate-3-phosphate EPSP: 5-enoylpyru vateshikimate-3-phosphate, $\mathrm{CHOR}$ : chorismate, $\mathrm{PPH}$; prephentate, (H)PPY: (hydroxyl)phenylpyruvate, 2-PE: 2-phenylethanol; PAA: phenylacetic acid; TYROSOL: tyrosol/para-hydroxyphenylethanol; PHPA: para-hydroxphenylacetate; TRP: tryptophan; TYR: tyrosine, PHE: phenylalanine

some non-Saccharomyces yeasts possess advantageous traits such as rapid growth, thermotolerance, salt tolerance, or the capacity to use a wider array of substrates [13]. The development of alternative hosts has previously been hampered by insufficient knowledge of cellular 
physiology, limited genetic tools, and a lack of experience of developing scaled bioprocesses. The rapid growth in the implementation of omics technologies, coupled with the development of synthetic biology [14], is helping address the first two of these problems, and several nonSaccharomyces yeasts are close to crossing the threshold to becoming credible first-choice hosts for defined applications.

One such yeast is the Crabtree-negative Kluyveromyces marxianus. It can tolerate temperatures over $50{ }^{\circ} \mathrm{C}$ and has the fastest growth rate of any eukaryote [15, $16]$. It can natively consume pentose sugars and disaccharides found in agricultural and dairy wastes, making these economic feedstocks for potential cell factories. The past decade has seen development of tools for its metabolic engineering such as CRISPR/Cas9 systems, libraries of regulatory elements, secretory peptides, and in vivo assembly protocols [17]. In parallel, K. marxianus has been successfully used as a platform to produce heterologous compounds such as short-chain alcohols, carotenoids and polyketides [18-20]. However, all the latter studies focus on the overexpression of heterologous enzymes or pathways and only a few studies attempt to engineer metabolism to improve production [21, 22]. With regards to aromatic compounds, $K$. marxianus has been evolved to overproduce phenylalanine and by extension the aromatic alcohol 2-phenylethanol (2-PE) via an enhanced Ehrlich pathway [23], but this has shed little light on appropriate metabolic engineering strategies. As its physiology relies more on respiration than that of S. cerevisiae, approaches to metabolically engineer $K$. marxianus to maximise precursor supply and product titres and yields can vary significantly from known ones in baker's yeast, especially since the production of aromatics is not a fermentative process.

In this work, we adopt a structured approach to metabolically engineer Kluyveromyces marxianus' AAA biosynthetic pathway to overproduce phenylalanine. Our strategy follows three stages. First, we alleviate feedback inhibition of the native shikimate pathway to establish AAA overproduction, followed by overexpression of the phenylalanine biosynthetic enzymes to enhance the production of phenylalanine. Second, we attempt to increase the supply of E4P and PEP to the shikimate pathway, first independently and then simultaneously, using native and heterologous enzymes. Finally, we redirect flux to phenylalanine synthesis alone by knocking down competing synthetic and degradative pathways. These strategies were assessed individually and in combination, and multiple different strategies aimed at similar goals were evaluated. Following this strategy allowed us to increase and redirect flux to the phenylalanine pathway so that it comprises up to $90 \%$ of the aromatic products synthesised.
In the course of our work, we track the production of 2-phenylethanol (2-PE), the Ehrlich degradation alcohol of phenylalanine as a proxy for the production of this amino acid. Our systematic investigation of engineering strategies for phenylalanine overproduction provide a roadmap for future metabolic engineers and synthetic biologists wishing to work with $K$. marxianus.

\section{Results}

\section{Establishing feedback-resistant phenylalanine biosynthesis in Kluyveromyces marxianus}

The aromatic amino acid biosynthetic and degradative pathways in K. marxianus share all steps in common with S. cerevisiae (Fig. 1; Additional file 1: Table S1), though there are some differences in numbers of paralogous genes. While $S$. cerevisiae has three phenylpyruvate decarboxylases Pdc5p, Pdc6p and Aro10p, K. marxianus only has a homologue to Aro10p and to Pdc5p, whose enzymatic activity is uncharacterised [24]. In contrast, besides homologues of the two aminotransferases found in S. cerevisiae (ARO8 and ARO9), sequenced K. marxianus genomes contain a third gene annotated as another putative aminotransferase [25]. The rational synthetic biology approach that we adopted involved the construction and analysis of over 60 strains in a complex matrix as we tested ways to enhance and optimise flux through the AAA biosynthetic pathway. A chart that shows the most important strains in this matrix is provided to aid understanding of the metabolic engineering approach and the strains generated in this study (Additional file 1: Fig. S1).

We began our engineering strategy by alleviating feedback inhibition of AAA synthesis in $K$. marxianus, a key step in establishing phenylalanine or tyrosine overproduction in yeast [9]. The $K$. marxianus enzymes (KmAro4p and KmAro7p) share $>75 \%$ sequence identity with their $S$. cerevisiae orthologues (Additional file 1: Table S1), thus we used knowledge of S. cerevisiae feedback resistant mutations as the basis of generating feedback resistant variants in $K$. marxianus enzymes. The substitutions K221L in KmAro4p ${ }^{\text {fbr }}$ and G141S in KmAro7p $\mathrm{p}^{\mathrm{fbr}}$ are equivalent to K229L and G141S mutations in the same enzymes in S. cerevisiae [9]. We initially overexpressed single copies of $\mathrm{KmARO}^{\mathrm{fbr}}$ and KmARO7 $7^{f b r}$ together in K. marxianus strain NBRC1777 on the centromeric plasmid pA1f/A4f-U. This strain, KmASR.004, produced detectable amounts of 2-PE and tyrosol (the Ehrlich alcohol derived from tyrosine), in the culture medium as a result of AAA overflow from the feedback resistant enzymes, along with significant amounts of shikimate. Approximately $1.2-1.4 \mathrm{mM}$, or $170 \mathrm{mg} \mathrm{L}^{-1}$ of each aromatic alcohol was produced after $48 \mathrm{~h}$ shake-flask culture in minimal medium at $30{ }^{\circ} \mathrm{C}$ along with $1.6 \mathrm{mM}$ shikimate, whereas the wild-type 
yeast did not produce any of these metabolites when grown for the same length of time (Fig. 2). This established that feedback resistant variants can be used in $K$. marxianus to alleviate feedback inhibition by both tyrosine and phenylalanine.

The next step was to create the centromeric plasmid pChor/A4f overexpressing both feedback-resistant alleles $\left(K m A R O 4^{f b r}\right.$ and $\left.K m A R O 7^{f b r}\right)$ and two other genes of the shikimate pathway, KmARO1 and KmARO2. The resulting strain, KmASR.009, did not show a significant increase in aromatic metabolite production relative to KmASR.004 (Fig. 2). Reasoning that this might be due to lack of metabolic pull downstream of Aro7 $\mathrm{p}^{\mathrm{fb} r}$, we further added two more genes, KmPHA2 and KmARO9, in a new single plasmid pAAA/Phe. Pha2p pushes flux coming from chorismate to phenylalanine, and Aro9p favours formation of phenylpyruvate (PPY) over phenylalanine, thereby facilitating our approach of using 2-PE as a readout for flux through this pathway (Fig. 1). Strain KmASR.010 with these additions increased 2-PE titres by $40 \%$ relative to KmASR.009, ultimately comprising nearly $70 \%$ of the measured Ehrlich metabolites (Fig. 2; Additional file 2: Supplementary Data 1). The increased pull of the remaining enzymes downstream of Aro7p also led to less shikimate being secreted, with the acid only comprising $20 \%$ of the total aromatic products as opposed to nearly $40 \%$ for ASR.004. At this stage, it appeared that all excess phenylalanine or tyrosine was being converted to 2-PE or tyrosol, respectively; the amino acids were neither detected as extracellular nor intracellular metabolites. We also did not detect any intracellular Ehrlich metabolites, suggesting that they were all secreted during growth.

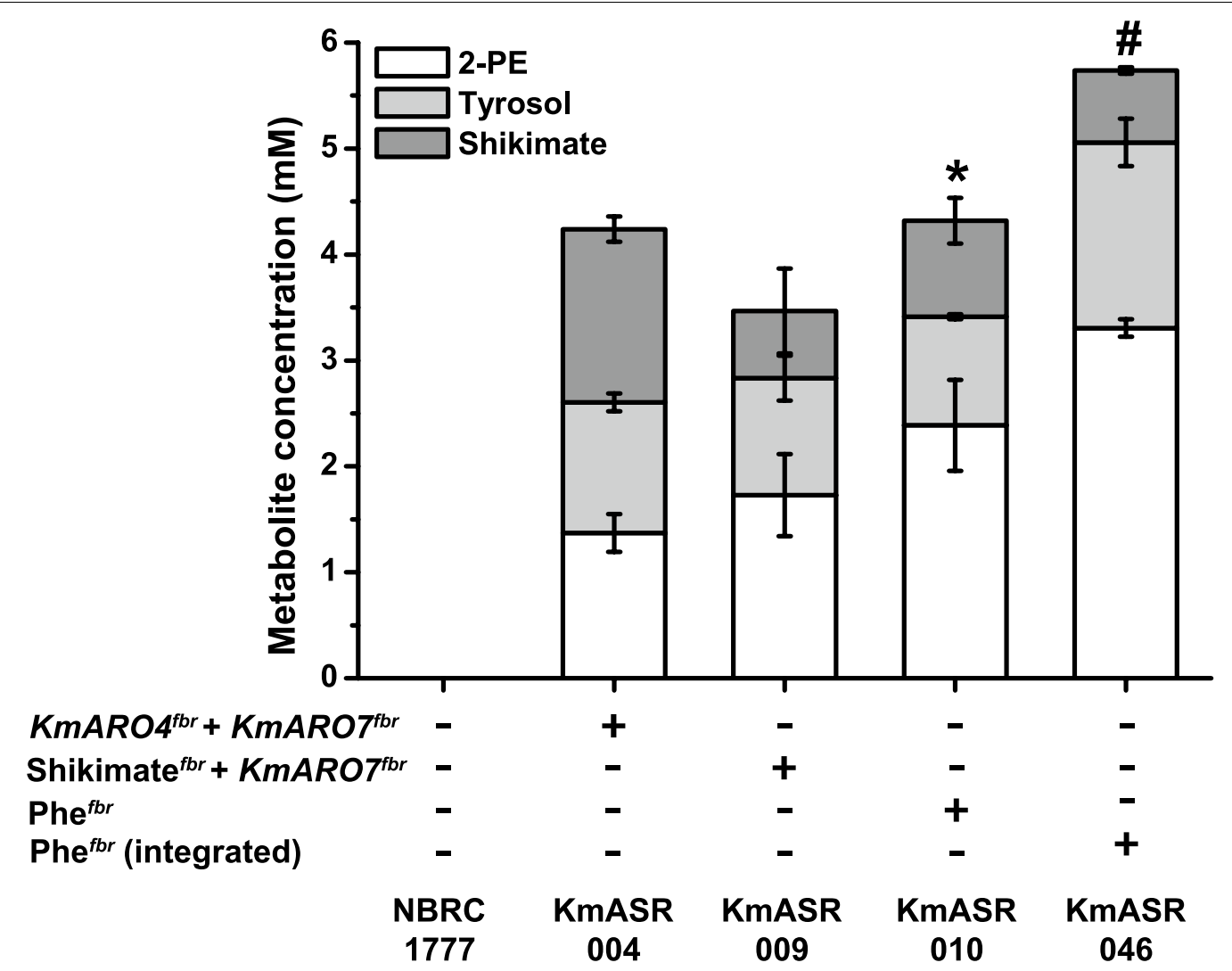

Fig. 2 Overexpression of a feedback-resistant phenylalanine biosynthetic pathway. The two feedback-resistant alleles of $\mathrm{KmARO4}$ and $\mathrm{KmARO7}$ constructed based on homology to S. cerevisiae are functional, with their episomal overexpression leading to the production of Ehrlich alcohols of phenylalanine (white) and tyrosine (grey) from the overflow of their increased synthesis, as well as excess shikimate (dark grey). All compounds were measured by HPLC in culture medium after $48 \mathrm{~h}$ growth at $30^{\circ} \mathrm{C}$ as described in the Methods. Subsequent co-expression of the shikimate (KmARO4 for $, K m A R O 1, K m A R O 2)+K m A R O 7^{f b r}$ and phenylalanine $\left(K m A R O 4^{\text {for }}+K m A R O 1+K m A R O 2+K m A R O 7^{f b r}+K m P H A 2+K m A R O 9\right)$ pathways increased their production and converted more shikimate to amino acids. Chromosomal integration of the pathway further improved phenylalanine and tyrosine production. Strains marked with an asterisk $\left(^{*}\right)$ show a significantly higher level of 2-PE relative to that produced by ASR.004 as determined by an independent $t$-test $(p<0.05)$. The data in the bar plots are the mean \pm s.d. of at least three biological replicates. All data plotted in this and following graphs can be found in Additional file 2: Supplementary Data 1 
We then decided to chromosomally integrate and overexpress the same pathway in a strain lacking the native alleles of ARO3, ARO4 and ARO7. Besides more stable expression, such a background would ensure that $K$. marxianus relies solely on the feedback-resistant phenylalanine/tyrosine pathway. Using CRISPR/Cas9, we inactivated KmARO4 and KmARO7 and then engineered a non-homologous-end-joining (NHEJ)-deficient background by inactivating the DNA ligase IV KmDNL4 by CRISPR/Cas9 [26, 27]. The use of NHEJ mutant backgrounds was necessary to ensure that only single copies of our constructs were integrated, as this was essential to accurately evaluate engineering strategies involving gene overexpression. Furthermore, targeted chromosomal integration is more successful in NHEJ mutants. We reconstructed the $\mathrm{Phe}{ }^{\mathrm{fbr}}$ pathway in an integrative vector pI6-AAA/Phe and integrated it at the KmARO3 locus to create strain KmASR.046. This strain lacks native Aro3p, Aro4p and Aro7p and is therefore fully reliant on the introduced enzymes for all AAA biosynthetic requirements. The combination of stable chromosomal integration of the pathway and loss of feedback-inhibited genes resulted in a final increase of nearly $40 \%$ in 2-PE production in the resultant strain KmASR.046 compared to plasmid-based expression in KmASR.010, with a final titre of $400 \mathrm{mg} \mathrm{L}^{-1} 2$-PE and $241 \mathrm{mg} \mathrm{L}^{-1}$ tyrosol $(3.31 \mathrm{mM}$ and 1.75 mM, Fig. 2).

\section{Increasing precursor supply to the shikimate pathway Increasing phosphoenolpyruvate supply to the shikimate pathway}

We next decided to investigate the best means to increase phosphoenolpyruvate (PEP) and erythrose-4-phosphate (E4P) supply to the shikimate pathway. PEP is a substrate in two steps of the shikimate pathway, required by Aro4p and Aro1p (Fig. 1). In yeast, PEP is produced by enolase (Eno1/2p) during glycolysis and PEP carboxykinase (Pck1p) during gluconeogenesis (Fig. 3a). In other species, PEP is also synthesised by PEP synthases (PEPS, EC 2.7.9.2) or pyruvate orthophosphate dikinase (PPDK, EC 2.7.9.1), enzymes that reversibly produce PEP from pyruvic acid [28]. In E.coli, the PEPS gene EcppsA has been frequently used to increase precursor supply to produce aromatic amino acid-derived compounds [29, 30]. Although EcppsA was unsuccessfully tested for a similar role in one K. marxianus strain [23], we decided to test it and several of these enzymes, initially in KmASR.004 and eventually in KmASR.046 with the full Phe ${ }^{\text {fbr }}$ (all steps from E4P/PEP to Phe) background. We overexpressed five codon-optimised PEPSes: EcppsA, an archaeal PEPS and three fungal PEPSes (Table 1). The fungal PEPSes were chosen based on their sequence homology to EcppsA, while the archaeal PEPS was chosen since it was reported to solely catalyse PEP formation [31]. We also chose to test a codon-optimised PPDK from Arabidopsis thaliana as it has a tenfold higher $K_{\mathrm{m}}$ for pyruvate than PEP as substrate, which could allow for efficient conversion of pyruvate to PEP [28]. We also included the native ENO1 from $K$. marxianus. Each of these genes was individually introduced into KmASR.004 and the effect on 2-PE production assessed (Fig. 3b). All three classes of enzyme were able to increase aromatic amino acid and shikimate production by providing more PEP for AAA synthesis. Among the heterologous enzymes, the PEPS from Aspergillus nomius (ASR.111) and PPDK from $A$. thaliana (ASR.108) were the best performing, both increasing 2-PE production to over $2 \mathrm{mM}$ (Fig. $3 \mathrm{~b}$ ). Overexpressing KmENO1 proved to be the most effective strategy to increase PEP, producing nearly $2.4 \mathrm{mM}$ 2 -PE, which represented an increase by over $70 \%$ relative to ASR.004 and was nearly identical to the titre produced using the entire Phe ${ }^{\mathrm{fbr}}$ pathway (KmASR.010 vs KmASR.109, Fig. 3b).

\section{Increasing erythrose-4-phosphate supply to the shikimate pathway}

The natural source for E4P is the non-oxidative branch of the pentose phosphate pathway (PPP) via cross-reactions from transketolase (Tkl1p) or transaldolase (Tal1p) (Fig. 3a). In S. cerevisiae, either or both of these enzymes are typically overexpressed to increase available E4P for AAA synthesis. More generally, metabolic engineering studies in S. cerevisiae revealed that partial or complete overexpression of different parts of the non-oxidative PPP can increase the production of AAA or shikimatederived products in combination with other modifications $[6,10,11]$. As $K$. marxianus has previously been reported to have a higher flux through the PPP than $S$. cerevisiae [32, 33], it was unclear whether overexpressing these enzymes would significantly improve E4P levels in $K$. marxianus. An alternative strategy tested in $S$. cerevisiae was to express a non-native fructose-6-phosphate phosphoketolase (Xfpk, EC 4.1.2.22), which converts fructose-6-phosphate to E4P and acetyl phosphate (Fig. 3a) [34]. We tested four phosphoketolases reported in the literature: three from Bifidobacterium breve, Lactococcus lactis and Rhodotorula graminis [34-36], as well as a phosphoketolase from Clostridium acetobuytlicum that favours xylulose-5-phosphate as a substrate over fructose-6-phosphate (Fig. 3c). Of the four phosphoketolases, only the Xfpk from B. brevis (Bbxfpk) increased the production of aromatic metabolites significantly, with 2-PE production increasing to over $2 \mathrm{mM}$ (KmASR.070). However, in all strains, this was accompanied by increased acetate production (Additional file 1: Fig. S2a) as well as slower growth compared to KmASR.004. In 


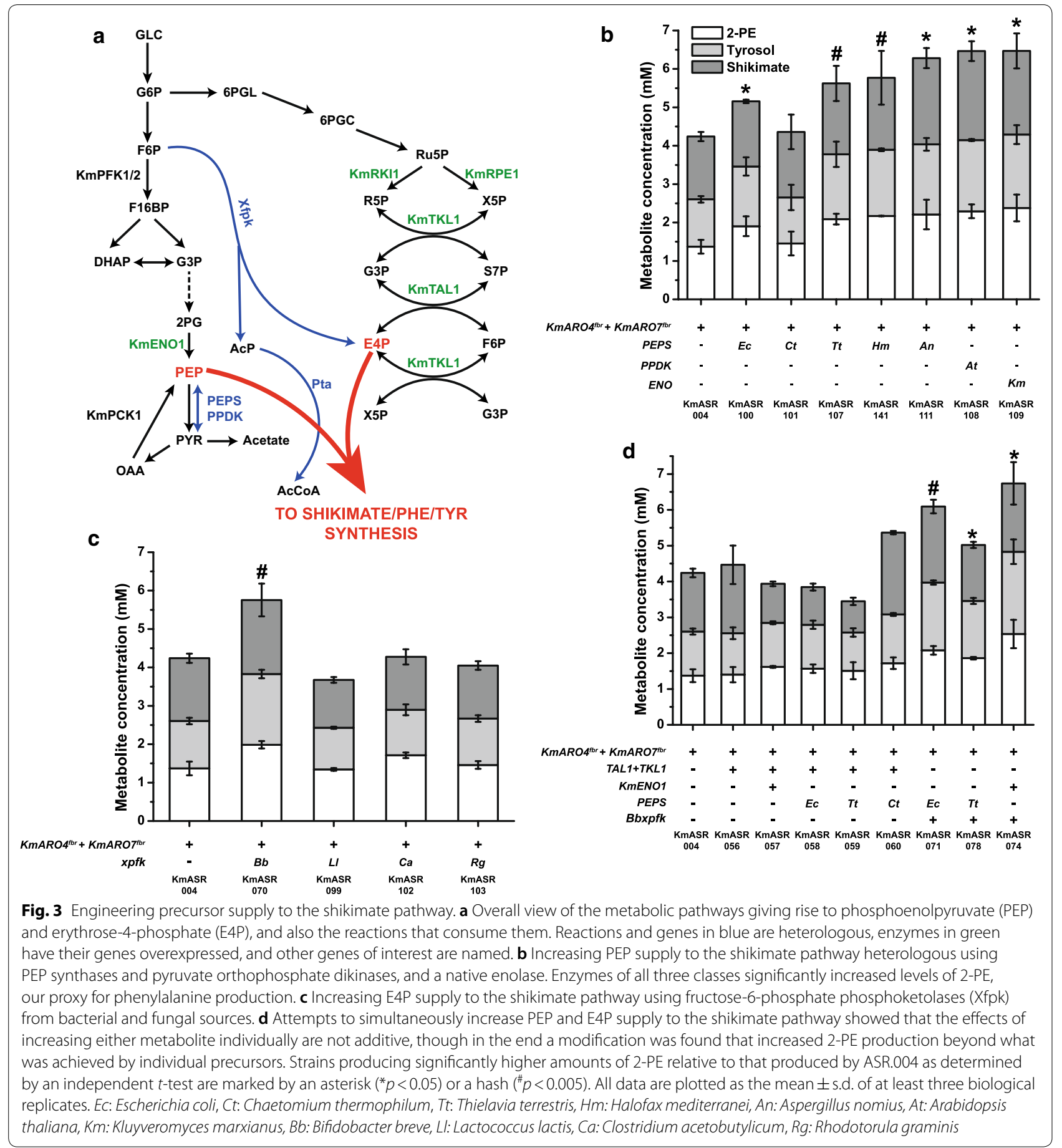

contrast, overexpressing KmTKL1 and KmTAL1 together (KmASR.056, Fig. 3d) did not significantly affect production of metabolites, with a slight increase in shikimate balanced overall by a decrease in tyrosine.

Finally, we decided to examine which combinations of enzymes best increased both precursors in KmASR.004 by coexpressing KmENO1 or different PEPSes alongside either Bbxfpk or the KmTKL1/TAL1 combination. Relative to our previous attempts to increase phenylalanine production one precursor at a time, only a combination of BbXfpk and KmEno1 further incrementally improved production (KmASR.074, Fig. 3d ), but this was not significantly higher than KmASR.070 or KmASR.109. This suggested that the combined effects of enzymes used to 
Table 1 Gene name, source organism and GenBank ID for heterologous genes used in this study. Nucleotide sequences are provided in Additional file 3: Supplementary Data 2

\begin{tabular}{lll}
\hline Gene/protein ID & Source organism & GenBank accession no \\
\hline EcPPSA/ECPEPS & Escherichia coli & P23538.5 \\
CtPEPS & Chaetomium thermophilum & EGS22573.1 \\
TtPEPS & Thielavia terrestris & AEO67530.1 \\
HmPEPS & Haloferax meditarranei & AFK18505.1 \\
AnPEPS & Aspergillus nomius & KNG90458.1 \\
AtPPDK & Arabidopsis thaliana & AEE83616.1 \\
Bbxfpk & Bifidobacteriumbreve & KND53308.1 \\
Llxfpk & Lactococcus lactis & AAK05600.1 \\
Caxfpk & Clostridium acetobutylicum & KHD36088.1 \\
Rgxfpk & Rhodotorula graminis & KPV77773.1 \\
Bspta & Bacillus subtilis & CAB15793.1 \\
Septa/eutD & Salmonella enterica & OAQ12105.1
\end{tabular}

increase PEP or E4P supply to the shikimate pathway were not purely additive. Furthermore, neither the PEPSes nor KmENO1, when coexpressed with KmTKL1 and
KmTAL1 in KmASR.004, significantly increased production (KmASR.057-KmASR.059, Fig. 3d ), nor did coexpressing PEPSes alongside $B b x f p k$ increase production over KmASR.070 (KmASR.070 vs KmASR.071, Fig. 3d).

\section{Integrating precursor supply with the phenylalanine biosynthetic pathway}

Our engineering of KmASR.004 identified strategies that could increase PEP and E4P supply to the shikimate pathway. We now wanted to test how these strategies would perform in a strain optimised for AAA production, which should exhibit downstream pull through the shikimate pathway. Unexpectedly, the two modifications that had been identified as the best performing in KmASR.004, overexpression of Bbxfpk (KmASR.082) by itself or with KmENO1 (KmASR.072), performed poorly and led to a reduction in titres of 2-PE (and tyrosol and shikimate) when introduced into KmASR.046 (Fig. 4a). One possible explanation was that the acetyl phosphate produced by Xfpk is further converted to acetate, lowering the cytosolic $\mathrm{pH}$ and increasing metabolic burden [37]. If the observed changes in 2-PE production are taken to be a proxy for a shifting metabolic burden, this

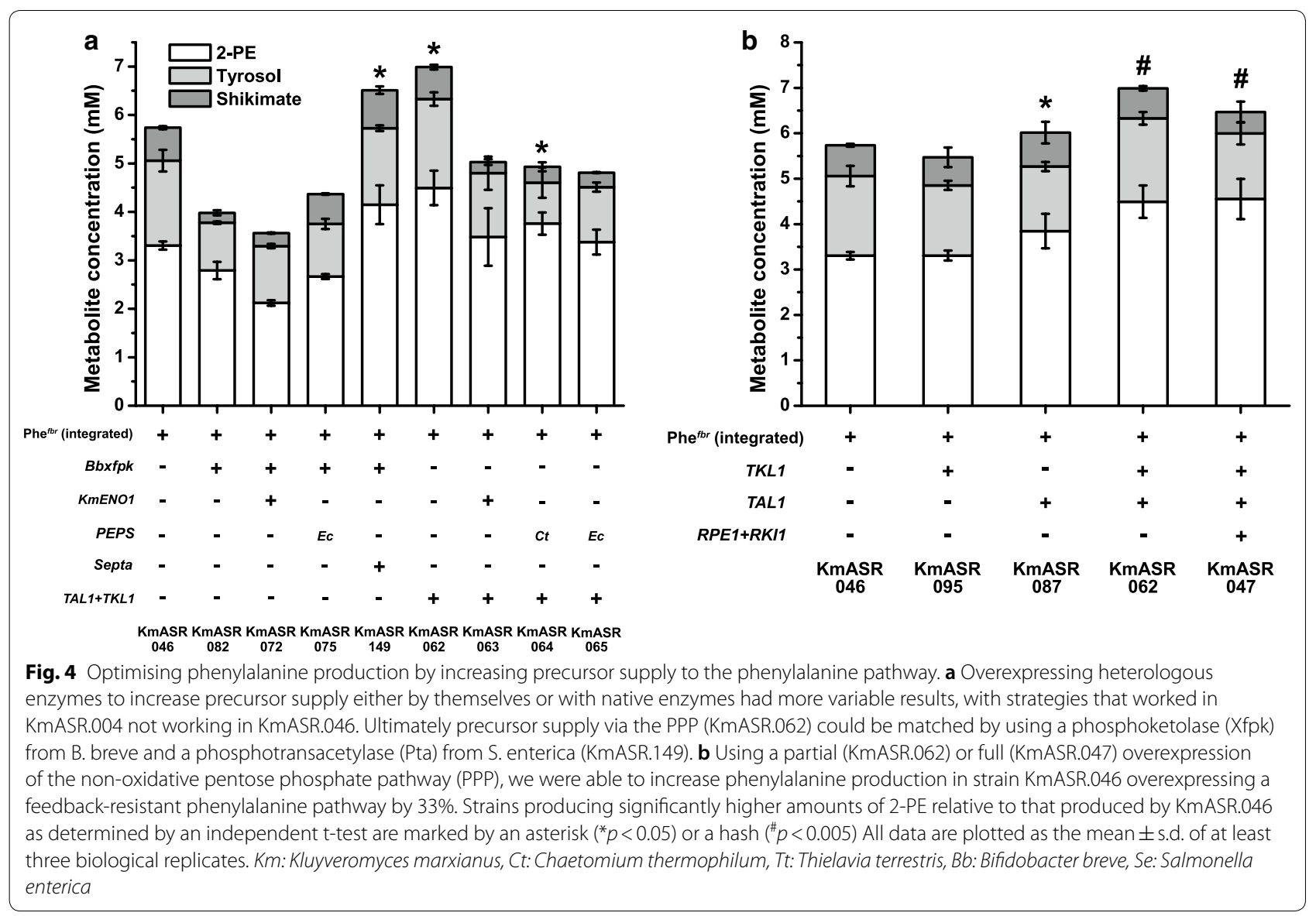


appears to be the case because the overexpression of a phosphotransacetylase (Pta, EC 2.3.1.8) from Salmonella enterica (Septa) [38] along with Bbxfpk alleviated the problem and generated strain KmASR.149 that produced over $4 \mathrm{mM}$ 2-PE. We also tested the effects of overexpression of enzymes of the non-oxidative branch of the PPP (Fig. 4b). While individual effects of KmTKL1 and KmTAL1 were weak, overexpression of both KmTKL1 and KmTAL1 (KmASR.062) or the entire non-oxidative branch of the PPP (KmASR.047) yielded $~ 4.5 \mathrm{mM} 2$ 2-PE. Combining overexpression of KmTKL1 and KmTAL1 (KmASR.062) with KmENO1 (KmASR.063) or bacterial PEPS (KmASR.064, KmASR.065) did not improve the titres of 2-PE (Fig. 4a), again illustrating that modifications are not simply additive.

Comparing all the derivatives of KmASR.046 tested, KmASR.047, KmASR.062 and KmASR.149 had the most improved production of phenylalanine, all producing up to $4.5 \mathrm{mM}$ (550 $\left.\mathrm{mg} \mathrm{L}^{-1}\right)$ 2-PE. Of these three, we decided to use KmASR.047 as the basis for integrating strategies to simultaneously increase E4P and PEP supply to phenylalanine biosynthesis. While the three mentioned strains produced approximately similar levels of 2-PE, tyrosol and shikimate, KmASR.047 began producing larger amounts of all aromatic metabolites within $24 \mathrm{~h}$ (Additional file 1: Fig. S3), compared to the other two strains, suggesting that this strain could be better optimised for faster bioprocesses to reach desired titres within a shorter fermentation time. However, the growth of the strains did not significantly differ, all having doubling times between 148 and $162 \mathrm{~min}$ when grown on minimal medium with glucose. We now took KmASR.047 as a base strain and considered approaches to improve PEP supply using the knowledge that we had gained. Because we had already found that interactions between pathways were complex, we also retested some of the enzymes that had not performed well previously (Fig. 5a). KmENO1 increased 2-PE production (KmASR.121), whereas overexpressing EcppsA or PPDK led to a significant decrease in the production of 2-PE (KmASR.120 and KmASR.142; Fig. 5a). Using the PEPSes from Aspergillus nomius (AnPEPS; KmASR.154) or Haloferax mediterranei (HmPEPS; KmASR.157) also reduced 2-PE titres but increased shikimate concentrations to $\sim 2 \mathrm{mM}$, compared to $0.46 \mathrm{mM}$ in KmASR.047, with a net increase in total aromatic metabolites (Fig. 5a). It may be that these enzymes, catalysing PEP formation reversibly, began to synthesise pyruvate from PEP and deplete levels of the latter in the metabolic background of

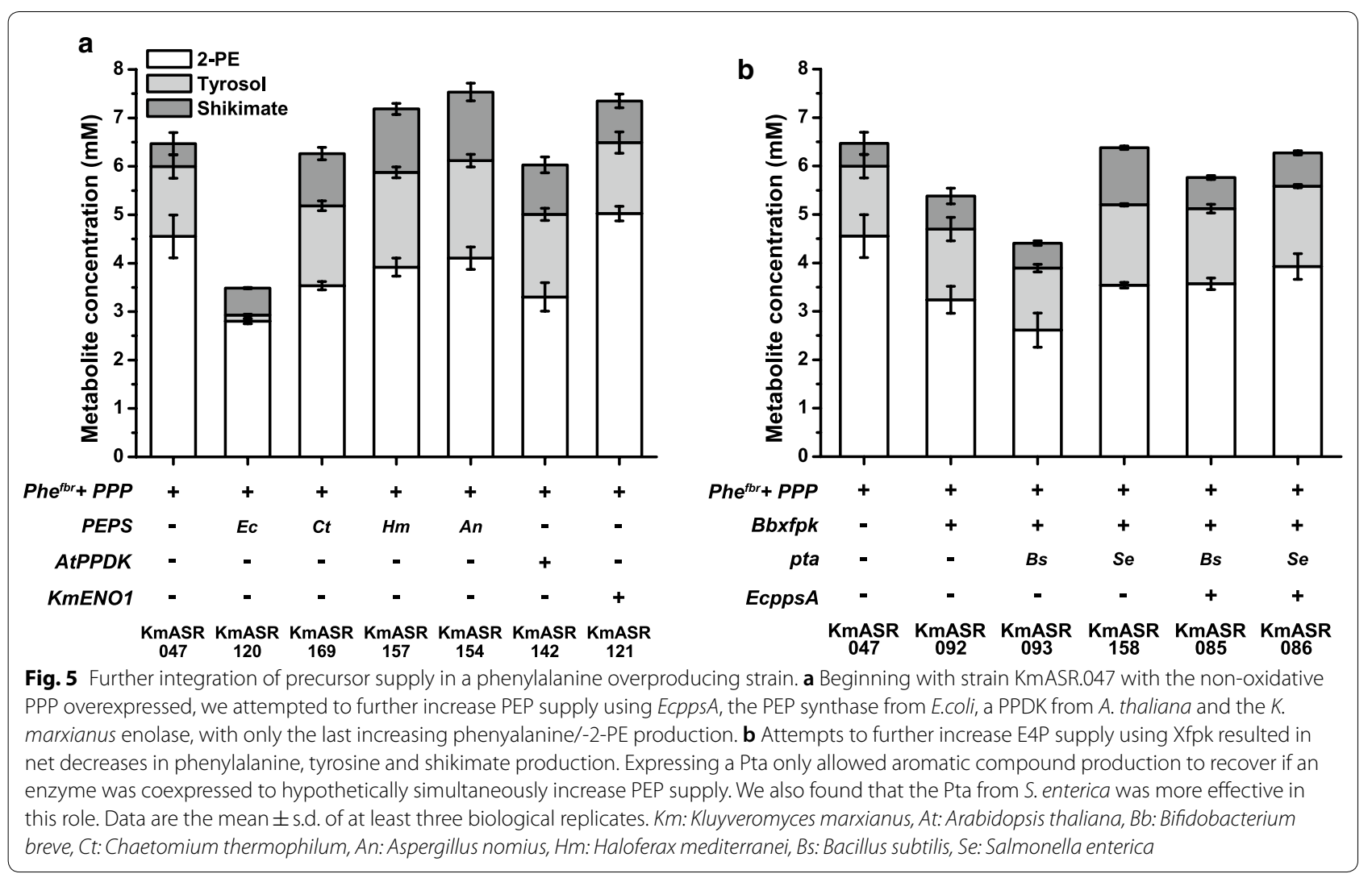


KmASR.047, leading to insufficient PEP to carry out the Aro1p-catalysed reaction (Fig. 1).

Further attempts to increase E4P supply in KmASR.047 using phosphoketolase were also unsuccessful (Fig. 5b). Overexpressing $B b x f p k$ in this strain decreased 2-PE production by nearly $30 \%$ to $3.24 \mathrm{mM}$ (KmASR.092) and, in contrast to the previous finding with KmASR.046, this effect was not alleviated by coexpressing Septa (KmASR.158). We also tested an additional PTA, Bspta from $B$. subtilis, but this also did not recover production (KmASR.093). An excess of E4P is known to inhibit bacterial DAHP synthase in the absence of PEP [39] and could be relieved by addition of excess PEP. We therefore coexpressed EcppsA alongside Bbxfpk and the two Ptas creating strains KmASR.085 and KmASR.086. While this did improve production over KmASR.093 and KmASR.0158, increasing production overall and converting more shikimate to 2-PE and tyrosol, 2-PE production was still less than KmASR.047. Taken together, these data suggest that careful balancing of the precursors to the phenylalanine biosynthetic pathway must be taken into consideration when increasing their availability.

\section{Optimisation of 2-phenylethanol production by knock-down of tyrosine production and aromatic aminotransferase deletion}

The 2-PE produced by the best-performing strains so far only amounts to approximately $70 \%$ of the total aromatic metabolites produced and $74 \%$ of the Ehrlich metabolites of phenylalanine and tyrosine. The third step of our engineering strategy is to knock down expression of the prephenate dehydrogenase KmTYR1 (Fig. 1). This enzyme converts prephenate to 4-hydroxphenylpyruvate, committing it to tyrosine synthesis: by reducing its expression as low as possible without creating a tyrosine auxotroph, more prephenate from Aro7p should be available for conversion to phenylpyruvate and phenylalanine. To achieve this, we substituted the native KmTYR1 promoter (TYR1pr) with one of two weaker promoters, GDH2pr and REV1pr, that we had previously characterised [27]. Both strains, KmASR.047kd and KmASR.047. $\mathrm{kd} 2$, increased the relative proportion of 2-PE to tyrosol, with the strongest effect seen when REV1pr (strain KmASR.047kd2) was used (Fig. 6). At this stage, 2-PE amounted to $89 \%$ of the Ehrlich metabolites and $83 \%$ of the aromatic metabolites measured. The decreased tyrosine production was still sufficient for prototrophy, and neither of the knock-downs created a significant growth defect in KmASR.047. Another strategy reported in the literature to increase 2-PE production is to delete the aromatic aminotransferase encoded by $A R O 8$ [40]. Inactivation of KmARO8 using CRISPR/Cas9 caused a $10 \%$ increase in all measured metabolites, with 2-PE titres increasing to $5.05 \mathrm{mM}$ (KmASR.112). We subsequently combined the two strategies in KmASR.047 to examine if these effects were additive for the production of 2-PE (KmASR.117). In KmASR.117, 2-PE production did not significantly increase $(6.31 \mathrm{mM}$ vs $6.25 \mathrm{mM}$ in KmASR.047kd2) but formed nearly 93\% of the total Ehrlich metabolites, as opposed to $89 \%$ and $82 \%$, respectively for the KmTYR1 knockdown and KmARO8 knockout alone (Fig. 6). The same modifications, when made to KmASR.062, resulted in a smaller increase in aromatic compound production resulting in the production of $5.2 \mathrm{mM} 2-\mathrm{PE}$ ( $634 \mathrm{mg} \mathrm{L}^{-1}$; Additional file 1: Fig. S4).

\section{Creation of a high 2-phenylethanol - producing strain by a synthesis of tested engineering strategies}

The three parts of our engineering strategy so far allowed us to establish feedback-resistant phenylalanine synthesis, optimise E4P supply for phenylalanine synthesis and then redirect metabolic flux within this pathway to create strain KmASR.117. However, lessons learned from other strategies to increase PEP and E4P supply led us to attempt to also increase precursor supply in this strain. Overexpressing KmENO1 in KmASR.117, led to a further increase in all aromatic compounds produced overall but with 2-PE only increasing by $4 \%$ (KmASR.127, Fig. 6). We then decided to take what were now our best strains and see what the effect of modifications for increase of E4P and/or PEP would be. We also included a feedback resistant allele of $K m A R O 3$ since an $A R O 3^{f b r}$ created in $S$. cerevisiae was found to aid aromatic amino acid synthesis when used in tandem with $A R O 4^{f b r}$ [41]. Based on the performance of previous strains, we hypothesised that KmAro3 $\mathrm{p}^{\mathrm{fbr}}$ would allow faster consumption of the increased E4P and PEP, avoiding their accumulation and diversion to other reactions. $K m A R O 3^{f b r}$ encoding a K222L mutation was overexpressed in KmASR.117 alongside Bbxfpk, Septa and EcppsA or KmENO1 to create strains KmASR.129 and KmASR.170, respectively. Of the two, the best performing strain was KmASR.129, which produced nearly $850 \mathrm{mg} \mathrm{L}^{-1}(6.95 \mathrm{mM}) 2-\mathrm{PE}$ in $48 \mathrm{~h}$, the highest titre so far, with a yield of $0.062 \mathrm{~mol}$ $2-\mathrm{PE} / \mathrm{mol}$ of glucose. Together, the final yield of aromatic metabolites (2-PE, tyrosol, shikimic acid) from KmASR.129 was $0.077 \mathrm{~mol} / \mathrm{mol}$ of glucose.

\section{Discussion}

K. marxianus is a promising next-generation yeast cell factory on account of its unique physiology, and has begun to be used for such applications. The same physiology and differences in metabolism to $S$. cerevisiae means that metabolic engineering approaches for this yeast require novel, rational approaches to best redirect its metabolism to generate precursors for valuable chemical 


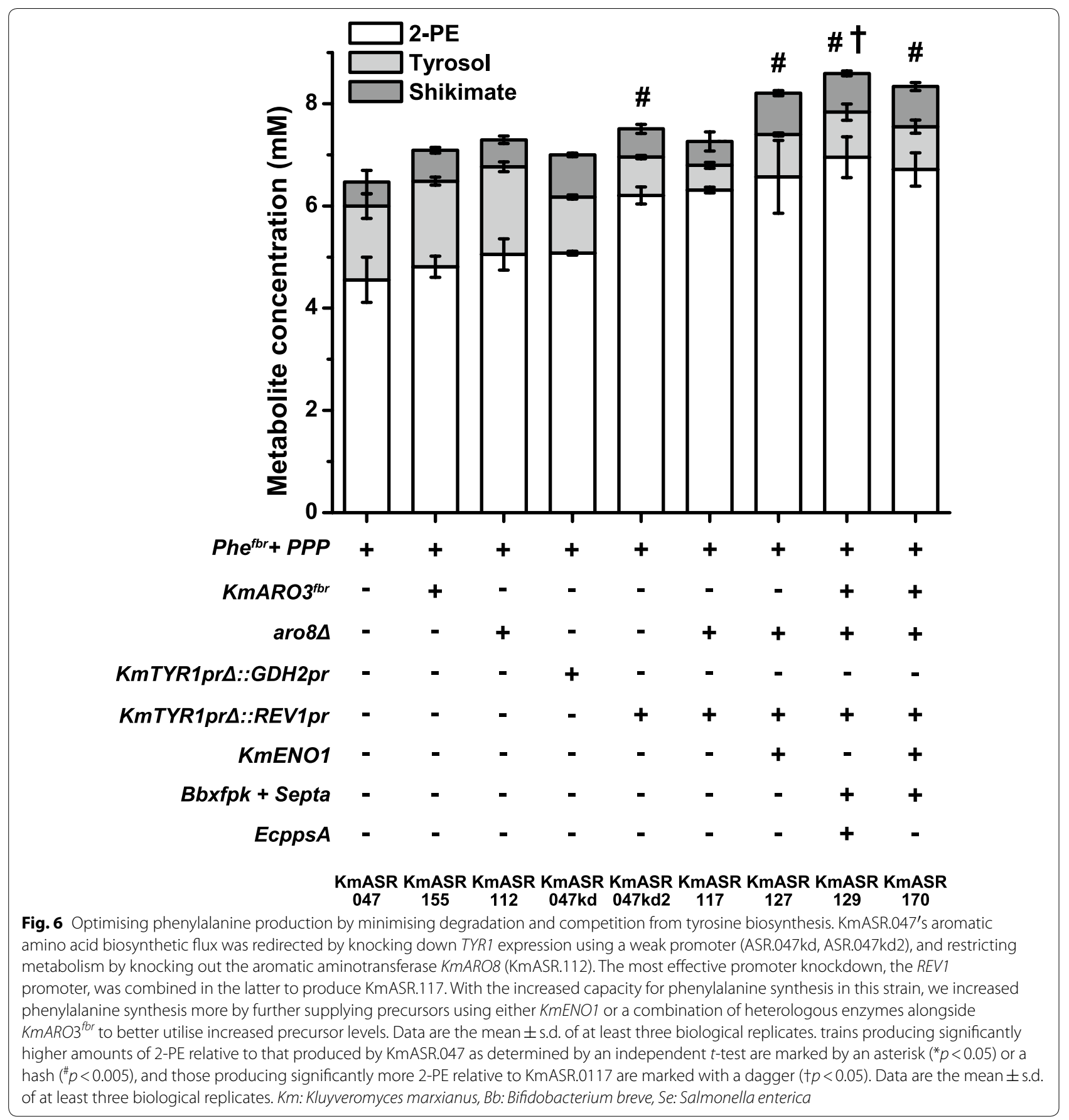

compounds. We implemented a three-step rational strategy to optimise a native biosynthetic pathway for overproduction and demonstrated its use for production of a commercially-relevant product de novo from synthetic growth medium. By (i) establishing phenylalanine overexpression via a feedback-resistant biosynthetic pathway, (ii) increasing precursor supply to the pathway, and (iii) knocking down competing pathways, reducing degradation and finally re-balancing precursor supply, our best-producing strains could synthesise $6.95 \mathrm{mM}$, or nearly $850 \mathrm{mg} \mathrm{L}^{-1}$, of 2-phenylethanol (2-PE) from glucose in $48 \mathrm{~h}$ flask cultivation. While we engineered K. marxianus for the production of 2-PE, relatively few modifications would be required to convert this strain to a phenylalanine production strain. The main modification would involve the inactivation of KmARO10 and 
$K m P D C 5$ to ensure the conversion of phenylpyruvate (PPY) to phenylalanine as opposed to 2-PE. This strain would be a platform for production of phenylalaninederived products.

Keeping K. marxianus' unique physiology in mind, we overexpressed heterologous enzymes that would make use of its high TCA cycle and PPP fluxes to increase precursor supply to the shikimate pathway [32, 33, 42]. By screening several PEP synthases, we were able to identify at least two, AnPEPS (in strains KmASR.108 and KmASR.154), and EcPEPS (in KmASR.129), that effectively convert pyruvate to PEP for the shikimate pathway in strains overexpressing a feedback resistant (fbr) phenylalanine biosynthetic pathway. Although a previous study suggested that EcPEPS could not function in this manner in $K$. marxianus [23], we found that the choice of PEPS and strain engineering played a role in the extent to which PEP synthase overexpression improved AAA or shikimate production. As some PEPSes are exclusively used by their host organisms for either gluconeogenesis or PEP synthesis [31, 43], a further investigation of more PEPSes with similar functions and kinetics may help us identify the best way to integrate this class of enzyme in yeast cell factories. In our efforts to increase E4P production, we overexpressed phosphoketolases that tap into K. marxianus' high fructose-6-phosphate levels and PPP flux $[33,37]$ to further boost available E4P. This strategy has recently been employed in other yeasts to increase the production of phenylalanine and tyrosine-derived metabolites with mixed results [12, 44-46]. While it was successful in K. marxianus, we found that an Xfpk's ability to increase the production of aromatic compounds is tempered by the extent of metabolic engineering on the AAA pathway. In contrast to previous studies in S. cerevisiae [45], we found that coexpressing a phosphotransacetylase (Pta) with $B b x f p k$ did improve production relative to when just Bbxfpk was expressed but that the choice of Pta mattered (KmASR.149, Fig. 4a; KmASR.158, Fig. 5b). Because as in S. cerevisiae, the overexpression of an Xfpk led to increased acetate secretion (Additional file 1: Fig. S2) [44], we suppose that expression of the Pta relieves metabolic burden. Further metabolomic studies on the impact of these combinations of enzymes on $K$. marxianus' metabolism, especially the TCA cycle and acetate metabolism, would be required to fully assess the metabolic burden that causes a decrease in 2-PE production (KmASR.082 vs KmASR.046, Fig. 4a; KmASR.092 vs KmASR.047, Fig. 5b).

Our final strain modifications highlighted the importance of balancing precursor supply and ensuring that precursors are efficiently utilised. This was especially apparent when optimal strategies to increase E4P and PEP varied across different scales of feedback-resistant
2-PE biosynthesis (Fig. 3, Fig. 5). Certain engineering strategies remain to be tried, such as mutating or deleting pyruvate kinase [12, 46], or utilising gluconeogenic enzymes to increase PEP. However, a combinatorial fine-tuning of gene expression, especially for PEP and E4P-supplying enzymes, could provide a more robust and scalable strategies to increase precursor supply since other modifications, such as the overexpression of $A R O 3^{f b r}$, only had an effect on 2-PE production when precursor supply was sufficiently increased for the enzyme to optimally function. Finally, as our best performing strains still leaked some shikimic acid from the pathway, overexpression of a bacterial shikimate kinase in addition to KmARO1 could be considered to ensure all the shikimate synthesised is used downstream and not secreted [12, 47]. In a broader sense, native enzymes in the shikimate and phenylalanine pathways could further be replaced by heterologous, more kinetically efficient homologues, or more multifunctional enzymes with equivalent activity [41].

One significant difference to $S$. cerevisiae engineering approaches we found was that whereas in S. cerevisiae overexpressing transketolase alone is sufficient to increase E4P levels, in $K$. marxianus, overexpression of both transketolase and transaldolase is required. While besides having a naturally high flux through the PPP, $K$. marxianus has also been reported to have high intracellular levels of fructose-6-phosphate [33], one of the substrates for transketolase but for the reverse reaction of interest; these high levels may inhibit the production of E4P by the overexpressed KmTKL1 and drive equilibrium in the opposite direction. By coexpressing it with KmTAL1, the two enzymes may be acting in tandem through their reactions, to ensure E4P production and avoiding the drawbacks of a single enzyme running in the reverse direction. Beyond this, overexpressing the rest of the pathway did not significantly improve phenylalanine production but provided a number of advantages such as a faster onset of production and more capacity for production once competing pathways were eliminated (Additional file 1: Figs. S3, S4).

The focus of our study was on developing a rational synthetic biology framework for building a $K$. marxianus chassis to overproduce aromatics and demonstrating its potential for strain engineering. With the caveat that different experimental set-ups make exact comparisons difficult, the titre $(6.95 \mathrm{mM})$ and yield $(62 \mathrm{mmol} / \mathrm{mol}$ glucose) of 2-PE achieved by our best-performing strain (KmASR.129) are within the range reported for other engineered de novo 2-PE producing yeasts: P. pastoris $(87 \mathrm{mmol} / \mathrm{mol}) ;$ Y. lipolytica $(97 \mathrm{mmol} / \mathrm{mol})$; and S. cerevisiae $(113 \mathrm{mmol} / \mathrm{mol})$ [12, 46, 48]. Interestingly, derivatives of a different $K$. marxianus strain (DMKU3-1042) 
were reported to produce up to $1.3 \mathrm{~g} 2-\mathrm{PE} \mathrm{L}^{-1}(10.7 \mathrm{mM})$ [23]. That study used a combination of rational engineering and laboratory evolution, which is a strategy that is routinely followed when moving from a prototype towards a production strain and process. Since we were interested in developing a platform rather than a bioprocess, we did not pursue this route, but it seems likely that the full potential of our $K$. marxianus strain for 2-PE production has not yet been reached. There are some notable differences between our findings with strain NBRC1777, and that study with strain DMKU3-1042, which illustrate the variation that strain selection can bring. Kim and colleagues report that overexpression of $A R O 10$ and $A D H 2$ from $S$. cerevisiae led to a significant increase in 2-PE production. In contrast, in our strain background, overexpressing the same genes in wild-type, KmASR.047 or KmASR.117 had only marginal effects (Additional file 1: Fig. S5). Based on these data and other indicators, it is likely that underlying differences in AAA metabolism between the two strains influence the effectiveness of particular engineering approaches, and more knowledge in this area is required. Future research into the comparative metabolomics of $K$. marxianus strains, especially with regard to amino acid and nitrogen metabolism, will help identify the most metabolically suited strains for specific applications.

\section{Conclusion}

In summary, we implemented a comprehensive synthetic biology strategy to build a $K$. marxianus strain for production of molecules derived from the aromatic amino acid phenylalanine. The potential of this chassis was demonstrated by constructing a cell factory that, starting from zero, produced almost $7 \mathrm{mM} 2$-PE from minimal mineral medium and glucose. Relatively few further modifications would be required to redirect metabolism for alternative products, or indeed to use tyrosine or tryptophan as the starting point. It would also be very interesting to apply our synthetic approach to the use of alternative carbon sources, in particular second generation feedstocks. Our study combined native and heterologous enzymes to increase the supplies of both phosphoenolpyruvate and erythrose-4-phosphate, and while not exhaustive, our findings reveal that balancing the precursors to shikimate and phenylalanine biosynthesis not only affects the amount but also the profile of aromatic metabolites produced. In two key aspects, the importance of synthetic biology approaches were very evident. First, it was necessary to try multiple versions of the same enzyme to identify the most suitable one for a particular reaction; and second, the effectiveness of any given modification was influenced in non-predictable ways by other modifications. Thus, a combinatorial, parallel approach is required to build high-performing strains. Although such strategies have been deployed with $S$. cerevisiae for some time, it was considered that the development of other yeasts was not sufficiently advanced to allow the same approach. With this study, we demonstrate that $K$. marxianus has reached the stage where synthetic biology is feasible and the steps that we followed may also serve as a roadmap for other non-traditional, or non-conventional, yeast cell factories.

\section{Materials and methods}

\section{Strains and media}

Kluyveromyces marxianus strain NBRC1777 from the Biological Resource Centre, NITE (NBRC; Tokyo Japan) was used as the base strain for all work in this study. For preliminary genome editing and knock-outs, strains were routinely grown in YPD broth $(2 \%$ yeast extract, $1 \%$ peptone and 2\% glucose; Formedium, Hunstanton, $\mathrm{UK})$, or synthetic drop-out (SD) medium with the relevant supplements $(0.5 \%$ ammonium sulphate, $0.19 \%$ yeast nitrogen base without amino acids or ammonia (Formedium), 2\% glucose and the relevant drop-out supplement according to the manufacturer's recommendations (Formedium)). When cells needed to be grown on solid media, the recipes above were supplemented with $2 \%$ agar (Formedium). For transformations involving CRISPR/Cas9, strains were grown on YPD agar with $200 \mathrm{mg} \mathrm{L}^{-1}$ hygromycin for selection. Bacterial transformations used E.coli DH5a grown in LB medium (1\% $\mathrm{NaCl}, 1 \%$ peptone, $0.5 \%$ yeast extract; Formedium) or LB agar $(+1.5 \%$ agar $)$ with the appropriate antibiotic (100 $\mathrm{mg} \mathrm{L}^{-1}$ ampicillin, $50 \mathrm{mg} \mathrm{L}^{-1}$ chloramphenicol or $50 \mathrm{mg} \mathrm{L}^{-1}$ kanamycin) as required. All antibiotics and reagents were purchased from Fisher Scientific (Dublin, Ireland) or Sigma-Alrdich/Merck (Haverhill, UK). All primers were obtained from Sigma-Aldrich.

\section{Plasmid construction}

All genes for the AAA biosynthetic pathway and non-oxidative PPP were amplified from genomic DNA from $K$. marxianus strain CBS6556 (Westerdijk Fungal Biodiversity Institute, Utrecht, The Netherlands) using Q5 HighFidelity Polymerase (M4092L, New England Biolabs (NEB), Ipswich, UK) with flanking nested BsaI (R0535L, NEB) and BsmBI (R0739L, NEB) restriction enzyme sites added by PCR. All synthetic heterologous genes were codon-optimised for S. cerevisiae and obtained from Twist Biosciences (San Francisco, U.S.A.). Sequences for these genes are available in Additional file 3: Supplementary Data 2. These respectively were used to clone the genes into storage plasmids (level I) by Golden Gate assembly with BsmBI and T7 DNA ligase (M0318L, NEB) for subsequent assembly into expression cassette-bearing 
plasmids (level II) using the hierarchical system based on the Yeast Toolkit standard [49]. Component vectors containing markers and connectors used to construct cloning vectors using this standard were a gift from John Dueber distributed through (Kit \# 1000000061, Addgene, Watertown, U.S.A.). The genes or gene fragments were cloned into the storage vector pYTK001 using a Golden Gate assembly with BsmBI. This plasmid contains a GFP cassette in its 'empty' state, allowing for green/white colony screening. Each plasmid's insert was verified by colony PCR following transformation into E.coli, and subsequent sequencing of the inserts of PCR-positive plasmids. For constructing feedback-resistant alleles of $K m A R O 4$ and $K m A R O 7$, the mutations were made to the genes during Golden Gate assembly by amplifying the gene as two fragments flanking the residue to be mutated. The mutated residue (K221L in KmARO4 and G141S in KmARO7) was included in the complementary overhangs for the BsmBI enzyme sites meant to join the fragments, so that on successful assembly of the fragments into a level I plasmid, the cloned product is the entire gene with the incorporated mutation. A similar strategy was used to eliminate internal BsaI or BsmBI sites that would otherwise interfere with Golden Gate assembly.

Level I plasmids for each gene and the desired promoter and terminator were then assembled into transcriptional units (TUs) by Golden Gate assembly with BsaI/T7 DNA ligase, which were then transformed into E.coli and verified by colony PCR using OneTaq Quick-Load Polymerase (M0486L, NEB) and restriction digestion by NotI (FD0596, Fisher Scientific). The K. marxianus promoters and terminators used for these plasmids have been described elsewhere [27]. Depending on their intended positions in a multi-TU plasmid, TUs in level II plasmids were cloned with direction-specific connectors flanked by BsmBI sites provided in the Yeast Toolkit to ensure directional assembly. Depending on the planned constructs, some TUs were cloned multiple times with different connectors. Finally, multiple TUs comprising pathways or parts of pathways were cloned into multi-TU plasmids by a Golden Gate assembly of the TU plasmids into drop-out expression vectors constructed in-house using BsmBI/T7 DNA ligase. All the pathway expression plasmids and cloning vectors are listed in Table 2. All level I and level II plasmids constructed for this study are listed in Additional file 1: Table S2, and the primers used to create them in Additional file 1: Table S3. More detailed information on Golden Gate assembly of the plasmids can be found in refs $[27,49]$.

\section{Production strain construction}

Strain background engineering

For plasmid-based experiments evaluating the overexpression of KmARO4 $4^{f b r}, K m A R O 7^{f b r}$, and the shikimate and phenylalanine pathways, we used the uracil auxotrophic strain KmASR.006 as a background. To create a background strain missing wild-type ARO3, ARO4 and $A R O 7$, we sequentially inactivated the genes using our CRISPR/Cas9 plasmid pUCC001, which contains a hygromycin resistance marker. As previously described, gRNAs targeting these genes were designed using sgRNA and cloned into pUCC001 by Golden Gate assembly [50, 51]. Starting with a ura ${ }^{-}$his $^{-}$auxotroph KmASR.008 we had previously constructed, we first inactivated KmARO4 and then KmARO7 sequentially. In each case, the CRISPR plasmid with the relevant gRNA target was transformed into KmASR.008 and selected for on YPD containing hygromycin. Up to 8 colonies were screened for mutations at KmARO4 or KmARO7 that caused a shift in the reading frame. After each successful gene inactivation, the strains were cured of the CRISPR plasmid by passaging an overnight culture of the confirmed mutant strain twice into YPD before being used for further genome editing. Mutant strains had their targeted genes sequenced again following curing to ensure the mutation was stable. The deletions provided us with strain KmASR.029 with an aro4 aro7 genotype. KmDNL4 was subsequently inactivated in this strain by CRISPR in a similar fashion to yield KmASR.039 to allow specific single integrations of pathway vectors via homologous recombination [26]. Previous characterization of NHEJdeficient backgrounds led us to select a dnl4- background due to lowest variation in gene expression of reporter inserts [27]. All integrations were performed in strains derived from KmASR.039 with the dn14-2 mutation to ensure the correct, targeted integration of single constructs. All strains used and constructed in this study are listed in Table 3, and all gRNA sequences used to inactivate genes are listed in Additional file 1: Table S4.

\section{Transformations and strain confirmation}

All transformations into $K$. marxianus were carried out by the lithium acetate/PEG method [52]. The expression vectors were either centromeric or integrative and were constructed as recommended for the Yeast Toolkit standard using a GFP drop-out cassette whose loss confirms successful assembly and allows for green/white colony screening of E.coli transformed with the Golden Gate assembly. All cloning vectors used in this work have been deposited in Addgene, with their part numbers listed in Additional file 1: Table S5. For strains establishing the overexpression of the feedback-resistant shikimate or phenylalanine pathway, plasmids of interest 
Table 2 Expression plasmids used in this study. Unless specified otherwise, all genes, promoters and terminators in the inserts are from $K$. marxianus

\begin{tabular}{|c|c|c|}
\hline Name & Contents/comments & Source \\
\hline pUCC001 & AaTEF1pr-SpCas9-ScPHO5t ScTDH3pr-HH-gRNA empty $-H D V-S c C Y C 1 t$ hphMX & [51] \\
\hline PMTU-DO-URA & Centromeric plasmid with GFP drop-out; ScURA3 KmARS/CEN7; available from Addgene (\#160004) & This work \\
\hline pMTU-DO-HIS & Centromeric plasmid with GFP drop-out; ScHIS3 KmARS/CEN7; available from Addgene (\#160005) & This work \\
\hline pl1-MTU-DO-HIS & Integrative plasmid with GFP drop-out targeting LAC4; ScHIS3; available from Addgene (\#160142) & This work \\
\hline pl6-MTU-DO-URA & Integrative plasmid with GFP drop-out targeting ARO3; ScURA3; available from Addgene (\#160030) & This work \\
\hline pl2-MTU-DO-G418 & Integrative plasmid with GFP drop-out, kanMX, targeting integration site I2 [27]; available from Addgene (\#160018) & This work \\
\hline pl4-MTU-DO-G418 & $\begin{array}{l}\text { Integrative plasmid with GFP drop-out, kanMX, targeting integration site I4 (Rajkumar et al., 2019); available from } \\
\text { Addgene (\#160215) }\end{array}$ & This work \\
\hline $\mathrm{pA} 1 \mathrm{f} / \mathrm{A} 4 \mathrm{f}-\mathrm{U}$ & 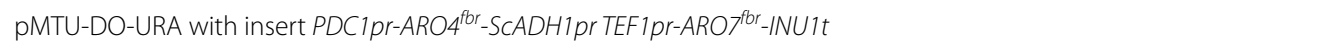 & This work \\
\hline pChor/A4f & 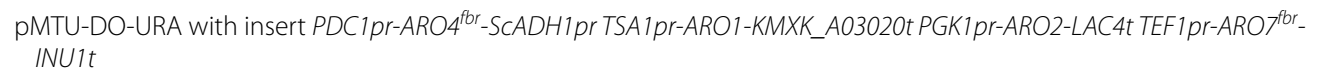 & This work \\
\hline pAAA/Phe & 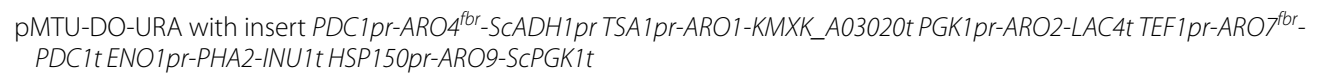 & This work \\
\hline pl6-AAA/Phe & $\begin{array}{l}\text { pl6-MTU-DO-URA with insert PDC1pr-ARO4 for-SCADH1prTSA1pr-ARO1-KMXK_A03020tPGK1pr-ARO2-LAC4t TEF1pr- } \\
\text { ARO7fbr-PDC1t ENO1pr-PHA2-INU1t HSP150pr-ARO9-SCPGK1t }\end{array}$ & This work \\
\hline pl1-P8A9T1 & pl1-MTU-DO-HIS with insert TEF1pr-TKL1-INU1t & This work \\
\hline pl1-P1A11T5 & pl1-MTU-DO-HIS with insert PGK1pr-TAL1-KMXK_A03020t & This work \\
\hline pl1-PPP & pl1-MTU-DO-HIS with insert TEF1pr-TKL1-INU1tPGK1pr-TAL1-KMXK_A03020t TDH3pr-RPE1-PDC1t TSA1pr-RKI1-ScPGK1t & This work \\
\hline pl1-TKAL & pl1-MTU-DO-HIS with insert TEF1pr-TKL1-INU1tPGK1pr-TAL1-KMXK_A03020t & This work \\
\hline pl1-TKAL-ENO & pl1-MTU-DO-HIS with insert TEF1pr-TKL1-INU1tPGK1pr-TAL1-KMXK_A03020tSSA2pr-KmENO1-SCADH1t & This work \\
\hline pl1-TKAL-A26 & pl1-MTU-DO-HIS with insert TEF1pr-TKL1-INU1tPGK1pr-TAL1-KMXK_A03020tSSA2pr-CtPEPS-SCADH1t & This work \\
\hline pl1-TKAL-A27 & pl1-MTU-DO-HIS with insert TEF1pr-TKL1-INU1tPGK1pr-TAL1-KMXK_A03020t SSA2pr-EcppsA-SCADH1t & This work \\
\hline PPPP-ENO & pMTU-DO-HIS with insert TEF1pr-TKL1-INU1tPGK1pr-TAL1-KMXK_A03020tSSA2pr-KmENO1-SCADH1t & This work \\
\hline PPPP-A26 & pMTU-DO-HIS with insert TEF1pr-TKL1-INU1tPGK1pr-TAL1-KMXK_A03020tSSA2pr-CtPEPS-SCADH1t & This work \\
\hline pPPP-A27 & pMTU-DO-HIS with insert TEF1pr-TKL1-INU1tPGK1pr-TAL1-KMXK_A03020tSSA2pr-EcppsA-ScADH1t & This work \\
\hline PPPP-A28 & pMTU-DO-HIS with insert TEF1pr-TKL1-INU1tPGK1pr-TAL1-KMXK_A03020tSSSA2pr-TtPEPS-SCADH1t & This work \\
\hline PTKAL & pMTU-DO-HIS with insert TEF1pr-TKL1-INU1tPGK1pr-TAL1-KMXK_A03020t & This work \\
\hline pP6B14T6-HIS & pMTU-DO-HIS with insert HSP150pr-Caxfpk-PDC1t & This work \\
\hline pP6B17T6-HIS & pMTU-DO-HIS with insert HSP150pr-Rgxfpk-PDC1t & This work \\
\hline pP6B18T6-HIS & pMTU-DO-HIS with insert HSP150pr-Bbxfpk-PDC1t & This work \\
\hline pP6B23T6-HIS & pMTU-DO-HIS with insert HSP150pr-LIxfpk-PDC1t & This work \\
\hline pP16A10T3-HIS & pMTU-DO-HIS with insert SSA2pr-KmENO1-SCADH1t & This work \\
\hline pP16A26T3-HIS & pMTU-DO-HIS with insert SSA2pr-CtPEPS-SCADH1t & This work \\
\hline PP16A27T3-HIS & pMTU-DO-HIS with insert SSA2pr-EcppsA-ScADH1t & This work \\
\hline pP16A28T3-HIS & pMTU-DO-HIS with insert SSA2pr-TtPEPS-SCADH1t & This work \\
\hline pP16A29T3-HIS & pMTU-DO-HIS with insert SSA2pr-AtPPDK-SCADH1t & This work \\
\hline pP16A37T3-HIS & pMTU-DO-HIS with insert SSA2pr-AnPEPS-SCADH1t & This work \\
\hline pP16А39T3-HIS & pMTU-DO-HIS with insert SSA2pr-HmPEPS-SCADH1t & This work \\
\hline pB18-A10 & pMTU-DO-HIS with insert HSP150pr-Bbxfpk-PDC1t SSA2pr-KmENO1-SCADH1t & This work \\
\hline $\mathrm{pB} 18-\mathrm{A} 27$ & pMTU-DO-HIS with insert HSP150pr-Bbxfpk-PDC1t SSA2pr-ppsA-SCADH1t & This work \\
\hline pB18-A28 & pMTU-DO-HIS with insert HSP150pr-Bbxfpk-PDC1t SSA2pr-TtPEPS-SCADH1t & This work \\
\hline pl4-P6B18T6 & pl4-MTU-DO-G418 with insert HSP150pr-Bbxfpk-PDC1t & This work \\
\hline pl4-pP16A10T3 & pl4-MTU-DO-G418 with insert SSA2pr-KmENO1-SCADH1t & This work \\
\hline pl4-P16A26T3 & pl4-MTU-DO-G418 with insert SSA2pr-CtPEPS-SCADH1t & This work \\
\hline pl4-P16A27T3 & pl4-MTU-DO-G418 with insert SSA2pr-EcppsA-SCADH1t & This work \\
\hline pl4-P16A29T3 & pl4-MTU-DO-G418 with insert SSA2pr-AtPPDK-SCADH1t & This work \\
\hline pl4-P16A37T3 & pl4-MTU-DO-G418 with insert SSA2pr-AnPEPS-SCADH1t & This work \\
\hline pl4-P16А39T3 & pl4-MTU-DO-G418 with insert SSA2pr-HmPEPS-SCADH1t & This work \\
\hline pl4-P20A30fT7 & pl4-MTU-DO-G418 with insert FBA1pr-KmARO3 ${ }^{\text {fbr-PGK1t }}$ & This work \\
\hline pl1-B18-A27 & pl1-MTU-DO-HIS with insert HSP150pr-Bbxfpk-PDC1t SSA2pr-EcppsA-SCADH1t & This work \\
\hline
\end{tabular}


Table 2 (continued)

\begin{tabular}{|c|c|c|}
\hline Name & Contents/comments & Source \\
\hline pl1-B38-HIS & pl1-MTU-DO-HIS with insert HSP150pr-Bbxfpk-PDC1t & This work \\
\hline pl4-B18-B15 & p14-MTU-DO-G418 with insert HSP150pr-Bbxfpk-PDC1t TEF1pr-BsPta-KMXK_A03020t & This work \\
\hline pl4-B18-B20 & p14-MTU-DO-G418 with insert HSP150pr-Bbxfpk-PDC1t TEF1pr-SePta-KMXK_A03020t & This work \\
\hline pl4-B18-B15-A27 & pl4-MTU-DO-G418 with insert HSP150pr-Bbxfpk-PDC1t TEF1pr-BsPta-KMXK_A03020t SSA2pr-EcppsA-ScADH1t & This work \\
\hline pl4-B18-B20-A27 & pl4-MTU-DO-G418 with insert HSP150pr-Bbxfpk-PDC1tTEF1pr-SePta-KMXK_A03020tSSA2pr-EcppsA-SCADH1t & This work \\
\hline pl2-B95-G418 & $\begin{array}{l}\text { pl2-MTU-DO-G418 with insert HSP150pr-Bbxfpk-PDC1t TEF1pr-SePta-KMXK_A03020t FBApr-KmARO3 fbr-PGK1t SSA2pr- } \\
\text { ppsA-SCADH1t }\end{array}$ & This work \\
\hline pl2-B78-G418 & $\begin{array}{l}\text { pl2-MTU-DO-G418 with insert HSP150pr-Bbxfpk-PDC1t TEF1pr-SePta-KMXK_A03020tFBApr-KmARO3 }{ }^{\text {for }} \text {-PGK1t SSA2pr- } \\
\text { KmENO1-SCADH1t }\end{array}$ & \\
\hline pl4-PEM & pl4-MTU-DO-G418 with insert ENO1pr-SCARO10-INU1t TEF1pr-SCADH2-PGK1t & This work \\
\hline pA8-KO-P9 & TYR1pr_US-REV1pr-TYR1pr_DS AmpR & This work \\
\hline pA8-KO-P11 & TYR1pr_US-GDH2pr-TYR1pr_DS AmpR & This work \\
\hline
\end{tabular}

were transformed into KmASR.008 and selected for on SD-ura agar. Subsequent plasmids containing genes to increase precursor supply (Additional file 1: Fig. S1) were transformed in the resultant strains and selected for on SD -ura- his agar. For the strain with the integrated feedback-resistant phenylalanine pathway KmASR.046, the pathway was cloned into the integrative vector pI6MTU-DO-URA targeting $K m A R O 3$, resulting in the vectors pI6-AAA/Phe. To ensure efficient targeting, $850 \mathrm{bp}$ sequences upstream and downstream of this gene were included as homology arms for this vector [53]. $1 \mu \mathrm{g}$ of this plasmid was digested with FastDigest SgsI (FD1894, Fisher Scientific), transformed into KmASR.039 and selected on SD-ura agar. Colonies appearing 3-5 days after transformation were screened by duplex colony PCR: one pair of primers checking integration of the pathway, priming upstream of the ARO3 locus and at the $5^{\prime}$ end of the construct, and another pair priming downstream of the ARO3 locus and at the $3^{\prime}$ end of the construct. KmASR.046 was selected from colonies positive for both bands. For the overexpression of genes increasing precursor supply in KmASR.046, genes of the non-oxidative pentose phosphate pathway or phosphoketolases/phosphotransacetlyase were cloned into the vectors pI1-MTU-DO-HIS or pI4-MTU-DO-HIS with a histidine auxotrophic marker targeting the LAC4 locus or the insertion site I4 on chromosome IV [27] respectively. Correct integration of the relevant construct was verified by colony PCR using two pairs of primers designed in a manner similar to those for checking the integration of pI6-AAA/Phe. The subsequent overexpression of genes in the resulting strains (Table 2, Additional file 1: Fig. S1) were done using the cloning vectors pI2-MTUDO-G418 and pI4-MTU-DO-G418 that target integration sites on chromosomes $\mathrm{V}$ and IV respectively and previously characterised by us [27]. Primer sequences for colony PCR verification are listed in Additional file 1: Table S2. The size of the integrated constructs varied from 3820 bp (pI4-P16A10T3, to overexpress KmENO1 in KmASR.121) to 18242 bp (pI6-AAA/Phe, to overexpress the Phe ${ }^{\mathrm{fbr}}$ pathway in KmASR.046 and derivatives). The efficiency of correct integration, as evaluated from the percentage of transformants with correct colony PCR products at loci I1, I2 and I4, varied from 25-100\%, and was affected by both construct size and integration locus. Efficiency was lower at the $A R O 3$ locus, varying from $6.25-50 \%$.

\section{Tyrosine knockdown by CRISPR/Cas9}

For marker-free replacement of TYR1pr, a gRNA target for Cas9 covering the first 13 bases of KmTYR1 and the first 7 upstream bases was identified by examining the start of the gene and its $5^{\prime}$ UTR [53]. These were used to construct gRNA plasmid pJ22. The REV1 or GDH2 promoters were cloned by Golden Gate assembly into a marker-free integrative vector flanked by 850 bp homology arms directly upstream and downstream of the gRNA sequence (while making sure the knock-down promoter was in-frame of KmTYR1) to yield plasmids pA8-KO-P9 and pA8-KO-P11 respectively. These plasmids were linearised with NotI and transformed into KmASR.047 alongside pJ22. Hygromycin-resistant colonies were screened for promoter replacement by colony PCR using a forward primer priming in the knock-down primers and a reverse primer at the end of KmTYR1. Promoter knockdown was $100 \%$ efficient and stable.

\section{Gene deletions}

gRNA targets for $K m A R O 8$ were identified and cloned into pUCC001 as previously described. However, as these were to be transformed into an NHEJ-deficient background, it was necessary to provide a linear repair 
Table 3 Strains used in this study

\begin{tabular}{|c|c|c|}
\hline Strain & Genotype & Source \\
\hline NBRC 1777 & Wild-type & $\begin{array}{l}\text { NITE } \\
\text { Biological } \\
\text { Resource } \\
\text { Center }\end{array}$ \\
\hline KmASR.006 & NBRC1777 ura3-1 & {$[27]$} \\
\hline KmASR.008 & NBRC1777 ura3-1 his3-2 & [27] \\
\hline KmASR.004 & KmASR.006 pA1f/A4f-U & This work \\
\hline KmASR.009 & KmASR.006 pChor/A4f & This work \\
\hline KmASR.010 & KmASR.006 pAAA/Phe & This work \\
\hline KmASR.021 & KmASR.008 pAAA/Phe pPPP & This work \\
\hline KmASR.029 & KmASR.008 aro4-1 aro7-1 & This work \\
\hline KmASR.039 & KmASR.029 dn/4-2 & This work \\
\hline KmASR.046 & KmASR.039 dn/4-2 aro3A:pl6-AAA/Phe & This work \\
\hline KmASR.047 & KmASR.046 dn/4-2 lac4A:pl1-PPP & This work \\
\hline KmASR.056 & KmASR.004 pTKAL & This work \\
\hline KmASR.057 & KmASR.004 pPPP-ENO & This work \\
\hline KmASR.058 & KmASR.004 pPPP-A27 & This work \\
\hline KmASR.059 & KmASR.004 pPPP-A28 & This work \\
\hline KmASR.060 & KmASR.004 pPPP-A26 & This work \\
\hline KmASR.062 & KmASR.046 dn/4-2 lac4ム:pl1-TKAL & This work \\
\hline KmASR.063 & KmASR.046 dn/4-2 lac4ム:pl1-TKAL-ENO & This work \\
\hline KmASR.064 & KmASR.046 dn/4-2 lac4ム:pl1-TKAL-A26 & This work \\
\hline KmASR.065 & KmASR.046 dn/4-2 lac4A: pl1-TKAL-A27 & This work \\
\hline KmASR.070 & KmASR.004 pP6B18T6 & This work \\
\hline KmASR.071 & KmASR.004 pB18-A27 & This work \\
\hline KmASR.072 & KmASR.046 dn/4-2 lac4ム: pl1-B18-A10 & This work \\
\hline KmASR.074 & KmASR.004 pB18-A10 & This work \\
\hline KmASR.075 & KmASR.046 dn/4-2 lac4A:pl1-B18-A27 & This work \\
\hline KmASR.047kd & KmASR.047 dn/4-2 TYR1pr $\triangle: G D H 2 p r$ & This work \\
\hline KmASR.047kd2 & KmASR.047 dn/4-2 TYR1prA:REV1pr & This work \\
\hline KmASR.078 & KmASR.004 pB18-A28 & This work \\
\hline KmASR.082 & KmASR.046 dn/4-2 144::pl4-P6B18T6 & This work \\
\hline KmASR.085 & KmASR.047 dn/4-2 144:pl4-B18-B15-A27 & This work \\
\hline KmASR.086 & KmASR.047 dn/4-2 144:p14- B18-B20-A27 & This work \\
\hline KmASR.087 & KmASR.046 dn/4-2 lac4A:pl1-P1A11T5 & This work \\
\hline KmASR.092 & KmASR.047 dn/4-2 14ム:p|4-P6B18T6 & This work \\
\hline KmASR.093 & KmASR.047 dn/4-2 144::p14-B18-B15 & This work \\
\hline KmASR.095 & KmASR.046 dn/4-2 lac4A:pl1-P8A9T1 & This work \\
\hline KmASR.099 & KmASR.004 pP6B23T6 & This work \\
\hline KmASR.100 & KmASR.004 pP16BA27T3 & This work \\
\hline KmASR.101 & KmASR.004 pP16BA26T3 & This work \\
\hline KmASR.102 & KmASR.004 pP6B14T6 & This work \\
\hline KmASR.103 & KmASR.004 pP6B17T6 & This work \\
\hline KmASR.107 & KmASR.004 pP16BA28T3 & This work \\
\hline KmASR.108 & KmASR.004 pP16BA29T3 & This work \\
\hline KmASR.109 & KmASR.004 pP16BA10T3 & This work \\
\hline KmASR.111 & KmASR.004 pP16BA37T3 & This work \\
\hline KmASR.112 & KmASR.047 dn/4-2 aro8 $\Delta 1$ & This work \\
\hline KmASR.115 & NBRC1777 dn/4-1 144::p|4-PEM & This work \\
\hline KmASR.117 & KmASR.112 dn/4-2 TYR1pr::REV1pr & This work \\
\hline
\end{tabular}

Table 3 (continued)

\begin{tabular}{|c|c|c|}
\hline Strain & Genotype & Source \\
\hline KmASR.119 & $\begin{array}{l}\text { KmASR.062 dn/4-2 TYR1pr::REV1pr } \\
\text { aro8 } \triangle 1\end{array}$ & This work \\
\hline KmASR.120 & KmASR.047 dn/4-2 144:pl4-P16BA27T3 & This work \\
\hline KmASR.121 & KmASR.047 dn/4-2 144:pl4-P16BA10T3 & This work \\
\hline KmASR. 122 & KmASR.047 dn/4-2 144::pl4-PEM & This work \\
\hline KmASR.123 & KmASR.062 dn/4-2 144::pl4-PEM & This work \\
\hline KmASR.127 & KmASR.117 dn/4-2 144:pl4-P16BA10T3 & This work \\
\hline KmASR.129 & KmASR.117 dn/4-2 I24:pl2-B95-G418 & This work \\
\hline KmASR.141 & KmASR.004 pP16BA39T3 & This work \\
\hline KmASR. 142 & KmASR.047 dn/4-2 144:pl4-P16BA29T3 & This work \\
\hline KmASR.149 & KmASR.046 dn/4-2lac44:pl1-B38-HIS & This work \\
\hline KmASR.154 & KmASR.047 dn/4-2 144:pl4-P16BA37T3 & This work \\
\hline KmASR.155 & KmASR.047 dn/4-2 144:pl4-P16BA37T3 & This work \\
\hline KmASR.157 & KmASR.047 dn/4-2 144:"pl4-P16BA39T3 & This work \\
\hline KmASR.158 & KmASR.047 dn/4-2 144::pl4-P20A30fT7 & This work \\
\hline KmASR.169 & KmASR.047 dn/4-2 144:pl4-P16BA26T3 & This work \\
\hline KmASR. 170 & KmASR.117 dn/4-2 124:pl2-B78-G418 & This work \\
\hline
\end{tabular}

fragment for homology-directed repair. These were designed by fusing two 80 bp sequences flanking a $412 \mathrm{bp}$ segment of the gene to be deleted. The repair fragment was ordered as two $90 \mathrm{bp}$ oligos with a $20 \mathrm{bp}$ region in common (the last $10 \mathrm{bp}$ of each flanking sequence). 50 pmol of each primer was then annealed and extended by Q5 polymerase in a short PCR reaction without a template to create a $160 \mathrm{bp}$ repair fragment, which was in turn transformed with the relevant gRNA plasmid targeting KmARO8 (pJA6). Hygromycin-resistant colonies were screened by colony PCR for the deletion by primers amplifying the entire gene; the difference in PCR product size relative to an intact gene indicated a successful deletion. Ultimately KmARO8 was knocked out in KmASR.047 to yield KmASR.112, and the deletion repeated out in the KmTYR1 knock-down strain ASR.047kd2 to create KmASR.117.

\section{Production strain cultivation and sampling}

The strain whose production was to be studied was grown $24 \mathrm{~h}$ in $5 \mathrm{~mL}$ of the appropriate SD medium (Formedium) at $30{ }^{\circ} \mathrm{C}$ in a shaking incubator set to $200 \mathrm{rpm}$. This culture was then diluted to an OD of 0.1 in $20 \mathrm{~mL}$ of minimal medium $\left(5 \mathrm{~g} \mathrm{~L}^{-1}\left(\mathrm{NH}_{4}\right)_{2} \mathrm{SO}_{4}, 3 \mathrm{~g} \mathrm{~L}^{-1} \mathrm{KH}_{2} \mathrm{PO}_{4}\right.$, $0.5 \mathrm{~g} \mathrm{~L}^{-1} \mathrm{MgSO}_{4} .7 \mathrm{H}_{2} \mathrm{O}, 20 \mathrm{~g} \mathrm{~L}^{-1}$ glucose with vitamins and trace elements, $\mathrm{pH} 6$ ) [54] in a $100 \mathrm{~mL}$ conical flask and grown at $30{ }^{\circ} \mathrm{C}$ and $200 \mathrm{rpm} .1 \mathrm{~mL}$ samples were taken at $48 \mathrm{~h}$ to sample extracellular metabolites. When more time points were sampled, a smaller volume of the culture medium was taken at each time point. After the $\mathrm{OD}$ of the culture was measured, the culture medium 
was centrifuged at 13,000 rpm for $10 \mathrm{~min}$ and the supernatant recovered and stored at $-20{ }^{\circ} \mathrm{C}$ until analysis. For the measurement of intracellular metabolites a pellet from $2 \mathrm{~mL}$ culture was boiled with $75 \%$ ethanol [55], the supernatant recovered and stored at $-20{ }^{\circ} \mathrm{C}$ until analysis. All data are from biological replicates within an experiment, but the findings were validated in independent experiments (technical replicates).

\section{Analytical methods}

Extra- and intra-cellular metabolite samples were thawed, vortexed, and transferred to screw-cap HPLC vials at an appropriate dilution. Shikimic acid, phenylalanine, tyrosine, 2-phenylethanol, tyrosol and para-hydroxyphenylacetic acid were analysed on an Agilent 1260 Infinity HPLC Infinity system with a Zorbax Eclipse Plus C18 column $(4.6 \times 150 \mathrm{~mm}, 3.5 \mu \mathrm{m}$; Agilent, Little Island, Ireland) operating at $40{ }^{\circ} \mathrm{C}$ using $20 \mathrm{mM} \mathrm{KH} \mathrm{PO}_{4}$ with $1 \%$ acetonitrile $(\mathrm{pH} 2) /$ acetonitrile as a solvent system at a flow rate of $0.8 \mathrm{~mL} \mathrm{~min}{ }^{-1}$ as previously described [2]. The elution gradient was as follows: acetonitrile was increased from $0-10 \%$ for the first $6 \mathrm{~min}$, then increased to $40 \%$ between 6 to $23 \mathrm{~min}$, and then switched to $100 \%$ $\mathrm{KH}_{2} \mathrm{PO}_{4}$ for 23 to $27 \mathrm{~min}$. All metabolites were detected using a VWD100 multi-wavelength detector measuring the absorbance at $200 \mathrm{~nm}$. Data were analysed using OpenLab CDS (Agilent). The elution times of the metabolites of interest were as follows: shikimic acid, $2.32 \mathrm{~min}$; tyrosine, $5.4 \mathrm{~min}$; phenylalanine, $7.5 \mathrm{~min}$; tyrosol/parahydroxyphenylethanol, $10.22 \mathrm{~min}$; para-hydroxyphenylacetic acid, $11.17 \mathrm{~min}$; 2-phenylethanol, $17.2 \mathrm{~min}$; phenylacetic acid; $17.86 \mathrm{~min}$. Standards for shikimic acid, phenylalanine, tryptophan, 2-phenylethanol and phenylacetic acid were obtained from Sigma-Aldrich, whereas standards for tyrosol and para-hydroxyphenylacetic acid were obtained from Fisher Scientific. Extracellular acetate concentration was determined with an Agilent 1200 HPLC system (Agilent Technologies, CA, USA) with a REZEX $8 \mu \mathrm{L} 8 \% \mathrm{H}+$ organic acid column $(300 \times 7.8 \mathrm{~mm})$ (Phenomenex, CA, USA) operating at $65 \circ \mathrm{C}$ using $0.01 \mathrm{~N}$ $\mathrm{H}_{2} \mathrm{SO}_{4}$ as a solvent at a flow rate of $0.6 \mathrm{~mL} \mathrm{~min}^{-1}$. Organic compounds were detected with a refractive index detector. All HPLC data for aromatic compounds in the graphs can be found in Additional file 2: Supplementary Data 1.

\section{Supplementary information}

Supplementary information accompanies this paper at https://doi. org/10.1186/s12934-020-01461-7.

Additional file 1: Table S1. The enzymes of the shikimate and phenylalanine/tyrosine biosynthetic pathways and the non-oxidative pentose phosphate pathway in Kluyveromyces marxianus. Table S2. Plasmids used to construct the pathway plasmids. All inserts are from Kluyveromyces marxianus unless specified otherwise. Table S3. Primers used in tTU study. Overhangs added by PCR that contain type IIS restriction enzyme sites for Golden Gate cloning are marked in boldface. Table S4. gRNA plasmids and their sequences used for genome engineering. The PAM is omitted. Table S5. Expression vectors constructed in the course of this study. Integration sites 12-14 are described in [2]. Figure S1. Strain construction flowchart. Engineering strategies and strains are coloured by the use of native or heterologous enzymes, and whether an improvement in 2-phenylethanol production was observed. Figure S2. Extracellular acetate production for strains using a KmASR.004 or b KmASR.046 as a base. Figure S3. Full expression of the non-oxidative pentose pathway allows for a faster production of 2-phenylethanol over short fermentation times. Figure S4. The effect of knocking down TYR1 expression and knocking out KMARO8 on KmASR.062 results in a smaller increase in 2-PE production than when the same modifications are made in KmASR.047. Figure S5. Overexpressing 2-PE producing genes form the Ehrlich pathway does not significantly improve 2-PE production in K. marxianus NBRC1777.

Additional file 2. Supplementary data 1.

Additional file 3. Supplementary data 2.

\section{Acknowledgements}

We thank Javier Varela, Joel Akinola, and Julia Bauer for help with plasmid construction, Else-Jasmijn Hassing for help with the HPLC protocol and Daniel Walsh for technical assistance with HPLC analysis. We also wish to thank Javier Varela, Else-Jasmijn Hassing, Jean-Marc Daran and other colleagues in the CHASSY project for advice and discussions.

\section{Authors' contributions}

ASR and JPM conceived the study and JPM supervised the research. ASR created the expression vectors, engineered the strains, and performed cultures to characterise the strain. ASR and JPM analysed the data, and wrote and revised the manuscript. All authors read and approved the final manuscript.

\section{Funding}

The authors were supported by the CHASSY project which received funding from the European Union's Horizon 2020 research and innovation programme under grant agreement No. 720824.

\section{Availability of data and materials}

All data generated or analysed during this study are included in this published article [and its supplementary information files]. Materials used in this study are available from Addgene or from the corresponding author on reasonable request.

Ethics approval and consent to participate

Not applicable.

\section{Consent for publication}

Not applicable.

\section{Competing interests}

The authors declare that they have no competing interests.

Received: 19 August 2020 Accepted: 27 October 2020

Published online: 11 November 2020

References

1. Liu Q, Liu Y, Chen Y, Nielsen J. Current state of aromatics production using yeast: achievements and challenges. Curr Opin Biotechnol. 2020:65:65-74.

2. Koopman F, Beekwilder J, Crimi B, van Houwelingen A, Hall RD, Bosch $D$, et al. De novo production of the flavonoid naringenin in engineered Saccharomyces cerevisiae. Microb Cell Fact. 2012;11:1-15.

3. Li Y, Li S, Thodey K, Trenchard I, Cravens A, Smolke CD. Complete biosynthesis of noscapine and halogenated alkaloids in yeast. Proc Natl Acad Sci U S A. 2018;115:E3922-31. 
4. Brown S, Clastre M, Courdavault V, O'Connor SE. De novo production of the plant-derived alkaloid strictosidine in yeast. Proc Natl Acad Sci U S A. 2015;112:3205-10.

5. Strucko T, Magdenoska O, Mortensen UH. Benchmarking two commonly used Saccharomyces cerevisiae strains for heterologous vanillin- $\beta$ glucoside production. Metab Eng Commun Elsevier. 2015;2:99-108.

6. Curran KA, Leavitt JM, Karim AS, Alper HS. Metabolic engineering of muconic acid production in Saccharomyces cerevisiae. Metab Eng. 2013:15:55-66.

7. Gottardi M, Reifenrath M, Boles E, Tripp J. Pathway engineering for the production of heterologous aromatic chemicals and their derivatives in Saccharomyces cerevisiae: bioconversion from glucose. FEMS Yeast Res. 2017:17:1-11.

8. Wendisch VF. Metabolic engineering advances and prospects for amino acid production. Metab Eng. 2019;58:17-34.

9. Luttik MAH, Vuralhan Z, Suir E, Braus GH, Pronk JT, Daran JM. Alleviation of feedback inhibition in Saccharomyces cerevisiae aromatic amino acid biosynthesis: Quantification of metabolic impact. Metab Eng. 2008;10:141-53

10. Suástegui M, Yu Ng C, Chowdhury A, Sun W, Cao M, House E, et al. Multilevel engineering of the upstream module of aromatic amino acid biosynthesis in Saccharomyces cerevisiae for high production of polymer and drug precursors. Metab Eng. 2017;42:134-44.

11. Mao J, Liu Q, Song X, Wang H, Feng H, Xu H, et al. Combinatorial analysis of enzymatic bottlenecks of I-tyrosine pathway by p-coumaric acid production in Saccharomyces cerevisiae. Biotechnol Lett. 2017;39:977-82.

12. Hassing E-J, de Groot PA, Marquenie VR, Pronk JT, Daran J-MG. Connecting central carbon and aromatic amino acid metabolisms to improve de novo 2-phenylethanol production in Saccharomyces cerevisiae. Metab Eng. 2019;56:165-80.

13. Radecka D, Mukherjee V, Mateo RQ, Stojiljkovic M, Foulquié-Moreno MR, Thevelein JM. Looking beyond Saccharomyces: the potential of nonconventional yeast species for desirable traits in bioethanol fermentation. FEMS Yeast Res. 2015;15:1-13.

14. Löbs AK, Schwartz C, Wheeldon I. Genome and metabolic engineering in non-conventional yeasts: current advances and applications. Synth Syst Biotechnol. 2017;2:198-207.

15. Lane MM, Morrissey JP. Kluyveromyces marxianus: A yeast emerging from its sister's shadow. Fungal Biol Rev. 2010;24:17-26.

16. Gombert AK, Madeira JV, Cerdán M-E, González-Siso M-I. Kluyveromyces marxianus as a host for heterologous protein synthesis. Appl Microbiol Biotechnol. 2016:100:6193-208.

17. Nurcholis M, Lertwattanasakul N, Rodrussamee N, Kosaka T, Murata M, Yamada M. Integration of comprehensive data and biotechnological tools for industrial applications of Kluyveromyces marxianus. Appl Microbiol Biotechnol. 2020;104:475-88.

18. Cheon Y, Kim JS, Park JB, Heo P, Lim JH, Jung GY, et al. A biosynthetic pathway for hexanoic acid production in Kluyveromyces marxianus. Biotechnol. 2014;182-183:30-6.

19. Lin YJ, Chang JJ, Lin HY, Thia C, Kao YY, Huang CC, et al. Metabolic engineering a yeast to produce astaxanthin. Bioresour Technol. 2017;245:899-905.

20. McTaggart TL, Bever D, Bassett S, Da Silva NA. Synthesis of polyketides from low cost substrates by the thermotolerant yeast Kluyveromyces marxianus. Biotechnol Bioeng. 2019;116:1721-30.

21. Cernak R, Estrella R, Poddar S, Skerker JM, Cheng Y-F, Carlson AK, et al. Engineering Kluyveromyces marxianus as a robust synthetic biology platform host. MBio. 2018;9:1-16.

22. Kong $X$, Zhang B, Hua Y, Zhu Y, Li W, Wang D, et al. Efficient L-lactic acid production from corncob residue using metabolically engineered thermo-tolerant yeast. Bioresour Technol. 2019;273:220-30.

23. Kim TY, Lee SW, Oh MK. Biosynthesis of 2-phenylethanol from glucose with genetically engineered Kluyveromyces marxianus. Enzyme Microb Technol. 2014;61-62:44-7.

24. Choo JH, Han C, Lee DW, Sim GH, Moon HY, Kim JY, et al. Molecular and functional characterization of two pyruvate decarboxylase genes, PDC1 and PDC5, in the thermotolerant yeastKluyveromyces marxianus. Appl Microbiol Biotechnol. 2018;102:3723-37.
25. Lertwattanasakul N, Kosaka T, Hosoyama A, Suzuki Y, Rodrussamee N, Matsutani M, et al. Genetic basis of the highly efficient yeast Kluyveromyces marxianus: complete genome sequence and transcriptome analyses. Biotechnol Biofuels. 2015;8:1-14.

26. Nambu-Nishida Y, Nishida K, Hasunuma T, Kondo A. Development of a comprehensive set of tools for genome engineering in a coldand thermo-tolerant Kluyveromyces marxianus yeast strain. Sci Rep. 2017;7:1-7.

27. Rajkumar AS, Varela JA, Juergens H, Daran JMG, Morrissey JP. Biological parts for Kluyveromyces marxianus synthetic biology. Front Bioeng Biotechnol. 2019;7:1-15.

28. Chastain CJ, Failing CJ, Manandhar L, Zimmerman MA, Lakner MM, Nguyen THT. Functional evolution of $C_{4}$ pyruvate, orthophosphate dikinase. J Exp Bot. 2011;62:3083-91.

29. Patnaik R, Liao JC. Engineering of Escherichia coli central metabolism for aromatic metabolite production with near theoretical yield. Appl Environ Microbiol. 1994;60:3903-8.

30. Juminaga D, Baidoo EEK, Redding-Johanson AM, Batth TS, Burd H, Mukhopadhyay A, et al. Modular engineering of L-tyrosine production in Escherichia coli. Appl Environ Microbiol. 2012;78:89-98.

31. Chen J, Mitra R, Zhang S, Zuo Z, Lin L, Zhao D, et al. Unusual Phosphoenolpyruvate (PEP) Synthetase-Like Protein Crucial to Enhancement of Polyhydroxyalkanoate Accumulation in Haloferax mediterranei Revealed by Dissection of PEP-Pyruvate Interconversion Mechanism. Appl Environ Microbiol. 2019;85:e00984-e1019.

32. Blank LM, Lehmbeck F, Sauer U. Metabolic-flux and network analysis in fourteen hemiascomycetous yeasts. FEMS Yeast Res. 2005;5:545-58.

33. Jung JY, Yun SH, Im DK, Lee J, Oh MK. 13C metabolite profiling to compare the central metabolic flux in two yeast strains. Biotechnol Bioprocess Eng. 2016;21:814-22.

34. Bergman A, Siewers V, Nielsen J, Chen Y. Functional expression and evaluation of heterologous phosphoketolases in Saccharomyces cerevisiae. AMB Expr. 2016. https://doi.org/10.1186/s13568-016-0290-0.

35. Evans CT, Ratledge C. Induction of xylulose-5-phosphate phosphoketolase in a variety of yeasts grown on d-xylose: the key to efficient xylose metabolism. Arch Microbiol. 1984;139:48-52.

36. Petrareanu G, Balasu MC, Vacaru AM, Munteanu CVA, lonescu AE, Matei I, et al. Phosphoketolases from Lactococcus lactis, Leuconostoc mesenteroides and Pseudomonas aeruginosa: Dissimilar sequences, similar substrates but distinct enzymatic characteristics. Appl Microbiol Biotechnol. 2014;98:7855-67.

37. Bergman A, Hellgren J, Moritz T, Siewers V, Nielsen J, Chen Y. Heterologous phosphoketolase expression redirects flux towards acetate, perturbs sugar phosphate pools and increases respiratory demand in Saccharomyces cerevisiae. Microb Cell Fact. 2019;18:1-13.

38. Brinsmade SR, Escalante-Semerena JC. The eutD gene of Salmonella enterica encodes a protein with phosphotransacetylase enzyme activity. J Bacteriol. 2004;186:1890-2.

39. Parker EJ, Bulloch EMM, Jameson GB, Abell C. Substrate deactivation of phenylalanine-sensitive 3-deoxy-D-arabino-heptulosonate 7-phosphate synthase by erythrose 4-phosphate. Biochemistry. 2001;40:14821-8.

40. Romagnoli G, Knijnenburg TA, Liti G, Louis EJ, Pronk JT, Daran JM. Deletion of the Saccharomyces cerevisiae ARO8 gene, encoding an aromatic amino acid transaminase, enhances phenylethanol production from glucose. Yeast. 2015:32:29-45.

41. Reifenrath M, Bauer M, Oreb M, Boles E. Bacterial bifunctional chorismate mutase-prephenate dehydratase PheA increases flux into the yeast phenylalanine pathway and improves mandelic acid production. Metab Eng Commun. 2018;7:1-9.

42. Sakihama Y, Hidese R, Hasunuma T, Kondo A. Increased flux in acetyl-CoA synthetic pathway and TCA cycle of Kluyveromyces marxianus under respiratory conditions. Sci Rep. 2019;9:1-7.

43. Zhang C, Anderson AJ. The gluconeogenic pathway in a soil Mycobacterium isolate with bioremediation ability. Curr Microbiol. 2013;66:122-31.

44. Liu Q, Yu T, Li X, Chen Y, Campbell K, Nielsen J, et al. Rewiring carbon metabolism in yeast for high level production of aromatic chemicals. Nat Commun. 2019;10:1-13. 
45. Guo W, Huang Q, Feng Y, Tan T, Niu S, Hou S, et al. Rewiring central carbon metabolism for tyrosol and salidroside production in Saccharomyces cerevisiae. Biotechnol Bioeng. 2020. https://doi.org/10.1002/bit.27370.

46. GuY, Ma J, Zhu Y, Ding X, Xu P. Engineering Yarrowia lipolytica as a chassis for de novo synthesis of aromatic-derived natural products and chemicals. ACS Synth Biol. 2020;9:2096-106.

47. Rodriguez A, Kildegaard KR, Li M, Borodina I, Nielsen J. Establishment of a yeast platform strain for production of $\mathrm{p}$-coumaric acid through metabolic engineering of aromatic amino acid biosynthesis. Metab Eng. 2015;31:181-8.

48. Kong S, Pan H, Liu X, Li X, Guo D. De novo biosynthesis of 2-phenylethanol in engineered Pichia pastoris. Enzyme Microb Technol. 2020;133:109459.

49. Lee ME, DeLoache WC, Cervantes B, Dueber JE. A highly characterized yeast toolkit for modular multipart assembly. ACS Synth Biol. 2015:4:975-86.

50. Xie S, Shen B, Zhang C, Huang X, Zhang Y. SgRNAcas9: A software package for designing CRISPR sgRNA and evaluating potential off-target cleavage sites. PLoS ONE. 2014;9:1-9.
51. Varela JA, Puricelli M, Montini N, Morrissey JP. Expansion and diversification of MFS transporters in Kluyveromyces marxianus. Front Microbiol. 2019;10:1-15.

52. Gietz RD. Yeast transformation by the LiAc/SS carrier DNA/PEG method. Methods Mol Biol. 2014;1163:33-44.

53. Inokuma K, Ishii J, Hara KY, Mochizuki M, Hasunuma T. Complete genome sequence of Kluyveromyces marxianus NBRC1777, a nonconventional thermotolerant yeast. Genome Announc. 2015;3:2-3.

54. Fonseca GG, Gombert AK, Heinzle E, Wittmann C. Physiology of the yeast Kluyveromyces marxianus during batch and chemostat cultures with glucose as the sole carbon source. FEMS Yeast Res. 2007;7:422-35.

55. Lai B, Plan MR, Averesch NJH, Yu S, Kracke F, Lekieffre N, et al. Quantitative analysis of aromatics for synthetic biology using liquid chromatography. Biotechnol J. 2017;12:1600269.

\section{Publisher's Note}

Springer Nature remains neutral with regard to jurisdictional claims in published maps and institutional affiliations.
Ready to submit your research? Choose BMC and benefit from:

- fast, convenient online submission

- thorough peer review by experienced researchers in your field

- rapid publication on acceptance

- support for research data, including large and complex data types

- gold Open Access which fosters wider collaboration and increased citations

- maximum visibility for your research: over 100M website views per year

At BMC, research is always in progress.

Learn more biomedcentral.com/submissions 